\title{
MlaFEDB displays flippase activity to promote phospholipid transport towards the outer membrane of Gram-negative bacteria
}

\author{
Gareth W. Hughes, Pooja Sridhar, Stephanie A. Nestorow, Peter J. Wotherspoon, Benjamin F. \\ Cooper, Timothy J. Knowles*
}

School of Biosciences, University of Birmingham, Birmingham, B15 2TT

${ }^{*}$ To whom any correspondence should be addressed: Timothy J Knowles, School of Biosciences, University of Birmingham, Birmingham, B15 2TT, U.K. E-mail: t.j.knowles@bham.ac.uk

\begin{abstract}
MlaFEDB is a Gram-negative inner membrane protein complex involved in the inter membrane trafficking of phospholipids. Originally proposed to transport phospholipids in a retrograde direction, recent evidence suggests MlaFEDB may actually export phospholipids from the inner membrane to the periplasmic carrier protein, $\mathrm{MlaC}$, potentially suggesting a role in either anterograde trafficking of phospholipids to the outer membrane or bidirectional phospholipid movement. MlaFEDB is part of the ABC transporter superfamily of proteins and has been shown to hydrolyse ATP through the cytoplasmic facing MlaF component. However, the movement of PLs from FEDB to MlaC has been shown to occur in an ATP independent fashion hence the role of ATP hydrolysis within this complex remains unclear. In this study we sought to elucidate the role of ATP and provide evidence to suggest MlaFEDB has flippase activity, utilising ATP hydrolysis to translocate phospholipids from the outer to the inner leaflet of the IM. We also show that in the absence of ATP MlaFEDB mediates the loading of $\mathrm{MlaC}$ with phospholipids directly from the inner leaflet only. Our data provides a novel role for MlaFEDB and presents a link between Mla driven phospholipid transport and ATP hydrolysis.
\end{abstract}

\section{Introduction}

Gram-negative bacteria are characterised by an asymmetric outer membrane (OM) of phospholipids (PL) and lipopolysaccharides (LPS) separated from the inner bacterial membrane (IM) by the periplasmic space containing a thin peptidoglycan layer (Malinverni and Silhavy 2009). This envelope acts as a robust permeability barrier to a vast array of environmental substances that would otherwise threaten bacterial viability, with much of this barrier provided by the OM (Kamio and Nikaido 1976, Nikaido 2005, Ruiz et al. 2006). The integrity of this membrane is maintained by localisation of LPS to the outer leaflet and PL to the inner leaflet. It is well established that transport of LPS to the outer leaflet is conducted by the Lpt machinery (Tran et al. 2008, Chng et al. 2010, Okuda et al. 2016). The mechanisms by which PL are transported to the OM are less well understood. Multiple investigations, however, have begun to implicate the maintenance of lipid asymmetry (Mla) pathway in having a 
role in this by ferrying PL across the periplasm to preserve PL homeostasis of the Gram-negative OM (Malinverni and Silhavy 2009, Sutterlin et al. 2016, Thong et al. 2016, Ekiert et al. 2017, Ercan et al. 2018, Hughes et al. 2019, Kamischke et al. 2019).

Malinverni \& Silhavy (2009) first proposed the Mla pathway as a retrograde transport system involved in the removal of PLs from the outer leaflet of the OM back to the IM through a series of protein complexes (Malinverni and Silhavy 2009); the lipoprotein MlaA at the OM, now identified as being associated with OmpF/C (Chong et al. 2015, Abellon-Ruiz et al. 2017), a periplasmically located soluble protein, MlaC (Ekiert et al. 2017, Hughes et al. 2019), and an inner membrane (IM) ATP binding cassette (ABC) transporter, MlaFEDB of stoichiometry 2:2:6:2 for MlaF, MlaE, MlaD and MlaB (Ekiert et al. 2017). From analysis of Mla knockouts, Malinverni \& Silhavy showed an increase in hepta-acylated LPS within the outer leaflet. This was believed to correspond with increased levels of PL present reported through the activity of the palmitoyl transferase PagP, known to synthesise hepta-acylated LPS using the sn-1 palmitoyl of outer leaflet PLs. This phenotype was shown to be recoverable with upregulation of the OM phospholipase protein PldA, known to dimerise and hydrolyze the sn-1 and sn-2 lipid tails of PLs in the presence of excess outer leaflet PLs. These experiments therefore suggested that Mla knockouts resulted in accumulation of outer leaflet PLs and determined that the Mla system had a role in their removal (Malinverni and Silhavy 2009). Further support for retrograde transport was given by Roier $e t$ al. (2016) who showed increased levels of IM vesicle (OMV) formation in Mla knockouts, suggesting that the accumulation of PL in the outer leaflet led to OMV formation (Roier et al. 2016). However more recently Kamischke et al. (2019) provided evidence to the contrary by showing that $\Delta m l a$ mutants have a decreased abundance of OM PLs and accumulate newly synthesised PL at the IM through direct quantification of membrane PLs (Kamischke et al. 2019). This was backed up by Ercan et al. (2019) who showed the movement of PLs from MlaD to MlaC (Ercan et al. 2018) whilst our laboratory, using membrane reconstituted MlaFEDB, observed unidirectional transport of PL to MlaC and the upregulation of ATPase activity only in the presence of substrate free MlaC (MlaC-apo) (Hughes et al. 2019), supporting a role not in retrograde transport but rather anterograde transport, the movement of PL towards the OM.

Although the key question regarding directionality has not yet been resolved, other questions also remain, in particular what is the role of ATP hydrolysis in the function of MlaFEDB complex? Previously we showed that MlaFEDB driven transport of PLs from the IM to MlaC in the periplasm occurs independently of ATP hydrolysis (Hughes et al. 2019). Despite this, as an ABC transporter, MlaFEDB functions as an ATPase (Thong et al. 2016). This discrepancy in function is difficult to reconcile, especially considering the large amounts of energy required to remove PLs from a bilayer (Marrink et al. 1998). Establishing a link between the ability of MlaFEDB to hydrolyse ATP and transfer PLs therefore represents an important area of research, especially given the potential role MlaFEDB plays in OM biogenesis.

In this study we therefore sought to address this and understand what processes are driven by ATP hydrolysis. Here we show that MlaFEDB has ATP driven flippase activity for all of the major PLs present within E.coli. We show flippase activity functions to translocate PLs from the outer to the inner leaflet of the IM and that in the absence of ATP MlaC accepts PL only from the inner leaflet through MlaFEDB. We propose that this flippase activity acts to maintain homeostasis within the IM by replenishing PLs removed from the IM by MlaC, further supporting the role of MlaFEDB in anterograde PL transport. 


\section{Results}

\section{Reconstitution of MlaFEDB in to fluorescently labelled phospholipid proteoliposomes}

The purification of N-terminally hexa-histidine tagged MlaFEDB in dodecylmaltoside (DDM) detergent, with the tag present on the N-terminus of MlaF has been described in detail previously (Hughes et al. 2019). This was incorporated in to liposomes containing E.coli polar lipids doped with the fluorophore nitrobenzoxadiazole on the headgroup of phosphatidylethanolamine (MlaFEDB:NBD-PE) via size exclusion chromatography (SEC) (Supplementary Fig. 1a). The mixing of NBD-PE with E.coli polar lipids prior to rehydration ensured NBD-PE was incorporated into both leaflets of the liposome bilayer. A single uniform peak was observed which was seen to homogeneously consist of MlaFEDB proteoliposomes which were found to be stable and consist of vesicular structures of $\sim 190 \mathrm{~nm}$ as measured by Dynamic light scattering (Supplementary Fig. 1b). Further use of SEC allowed us to generate NBD-PE labelled E.coli polar lipid liposomes devoid of protein, here termed PL:NBD-PE, and as such could be used as control samples to compare differences in NBD-PE leaflet distribution. NBD-PE was chosen initially, as PE is the predominant PL in E.coli, making up $~ 70 \%$ of the total PL pool.

\section{MlaFEDB preferentially orientates within liposomes}

In order to begin to probe the role of ATP in MlaFEDB function, it was important to elucidate the relative orientations of MlaFEDB within the proteoliposome, e.g. either MlaF or MlaD outward facing. To ascertain this we used proteinase $\mathrm{K}$ treatment in combination with western blotting against the hexa-histidine tag at the $\mathrm{N}$ terminus of MlaF to ascertain the ratio of outward MlaF facing to inward facing complex (Fig. 1a). It was found that approximately $90 \%$ of the complex preferentially oriented itself with the MlaF ATP binding domain outward facing (accessible to ATP), with $\sim 10 \%$ orientating such that MlaD faces outward (accessible to MlaC). However, as both populations existed, unidirectional study could be achieved by either the addition of ATP or the addition of MlaC to the outside of the proteoliposome (Fig. 1b).

\section{MlaFEDB mediates the transbilayer movement of phospholipids.}

It is well established that another Gram-negative IM ABC transporter, MsbA, functions as a flippase and is involved in Lipid A transport (Eckford and Sharom 2010). As a functionally similar IM complex, we hypothesised that MlaFEDB may also display the ability to translocate lipids between leaflets of the IM to maintain IM integrity following PL removal. To investigate this potential flippase activity we used a modified lipid flippase assay based on the work of Eckford and Sharom (Eckford and Sharom 2005) to quantify changes in the distribution of fluorescently labelled PLs across proteoliposomal bilayers in response to different treatments. It has already been well established that the Mla pathway shows specificity for the PL tail rather than head group (Thong et al. 2016, Ekiert et al. 2017, Hughes et al. 2019), we hypothesised that the presence of a fluorescent tag would therefore minimally affect activity. Briefly, total fluorescence could be measured at an emission wavelength of $536 \mathrm{~nm}$, with readings equating to the levels of NBD-PE in both the inner and outer leaflets of the membrane. Through the use of the membrane impermeable, fluorescence-quenching agent, sodium dithionite, it is then possible to directly measure inner leaflet fluorescence emitted from NBD-PE alone. Final treatment with Triton X-100 solubilises the proteoliposome, promoting quenching of the inner leaflet and allowing a 
determination of background fluorescence. As such, it is possible to directly measure the levels of NBD-PE within both the inner and outer leaflets of the membrane.

To ascertain if MlaFEDB performs flippase activity, MlaFEDB:NBD-PE was incubated at $25{ }^{\circ} \mathrm{C}$ in the presence or absence of ATP for $1 \mathrm{hr}$. As ATP is unable to cross the liposomal bilayer and was added after proteoliposome formation, any change in distribution would result from the population of MlaFEDB with the MlaF component outward facing only (Fig. 1b). Fluorescence changes observed across the membrane were then measured. In the absence of ATP, NBD-PE was seen to be evenly distributed across the inner and outer leaflets of the MlaFEDB:NBD-PE proteoliposome, with $\sim 48 \%$ NBD-PE present in the inner leaflet following dithionite quenching (Fig. 2a), suggesting that in the absence of ATP no flippase activity was observed, consistent with proteoliposome PL distribution observed in other systems (Eckford and Sharom 2010). However, in the presence of ATP we observed a significant shift in this distribution, with the percentage of inner leaflet NBD-PE decreasing to $20 \%$ (Fig. 2a). This large change in fluorescence distribution is highly indicative of a MlaFEDB driven ATP dependent translocation of NBD-PE from the inner to the outer leaflet of the proteoliposome.

To ensure that any observed PL translocation is subject to flippase mediated activity and not due to the intrinsic flip-flop of PLs, PL:NBD-PE was also incubated at $25{ }^{\circ} \mathrm{C}$ in the presence or absence of ATP for $1 \mathrm{hr}$. Irrespective of the presence of ATP PL:NBD-PE liposomes were seen to display similar levels of NBD-PE distributed across the inner and outer leaflets of the liposome, with $\sim 47 \%$ NBD-PE present in the inner leaflet following dithionite quenching (Fig. 2b). These levels are comparable to those observed in untreated MlaFEDB:NBD-PE proteoliposomes.

To further assess the ATP dependence of MlaFEDB driven NBD-PE translocation we performed translocation assays in the presence of increasing amounts of ATP $(0-1 \mathrm{mM})$, with the flippase activity measured over the course of an hour. Translocation of NBD-PE from the inner to the outer leaflet of the proteoliposome was seen to occur in an ATP concentration dependent manner, with increasing levels of ATP resulting in increased movement of NBD-PE to the outer proteoliposomal leaflet (Fig. 2c \& Supplementary Fig. 2).

\section{MlaFEDB flippase activity is dependent upon ATP hydrolysis.}

Through the use of non-hydrolysable ATP analogues, GTP, and ATPase null mutants we were able to further examine the ATP-dependence of the MlaFEDB driven flippase activity.

To test whether flippase activity is stimulated by the binding of ATP or by the hydrolysis of ATP we analysed the flippase activity of MlaFEDB:NBD-PE proteoliposomes in the presence of ATP $\gamma \mathrm{S}$, a nonhydrolysable analogue of ATP. Previously we have shown MlaFEDB was still able to retain PL transport function, transferring PLs to MlaC in the presence of ATP $\gamma \mathrm{S}$ (Hughes et al. 2019). Here in the presence of ATP $\gamma \mathrm{S}$ we saw no significant change in the percentage of fluorescent PL (47\%) within the inner leaflet, a level comparable to the quantity of fluorescent PL observed in the absence of ATP (47\%), suggesting that flippase activity does not occur.

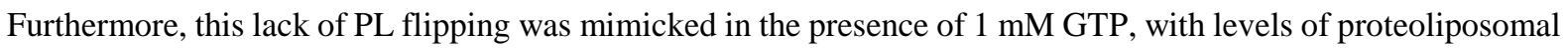
inner leaflet fluorescence remaining at around $47 \%$ in both the presence and absence of GTP (Fig. 3a \& Supplementary Fig. 3).

To further probe the functional independence of MlaFEDB driven PL transport and flippase activity we utilised the MlaFEDB ATPase null mutant, MlaF $\mathrm{K}_{47 \mathrm{R}} \mathrm{EDB}$, a non-functional MlaF walker A motif mutation (Thong et al. 2016). Here we purified $\mathrm{MlaF}_{\mathrm{K} 47 \mathrm{R}} \mathrm{EDB}$ to homogeneity (Supplementary Fig. 4a) and again showed 
its lack of inherent ATPase activity (Supplementary Fig. 4b). Through binding to metal chelate resin, we were able to stably reconstitute $\mathrm{MlaF}_{\mathrm{K} 47 \mathrm{R}} \mathrm{EDB}$ into E.coli polar lipid liposomes, thus allowing us to probe lipid transport through incubation with MlaC-apo and analysis via thin layer chromatography, as we have shown previously for

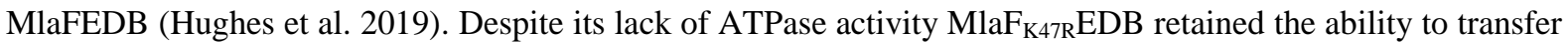

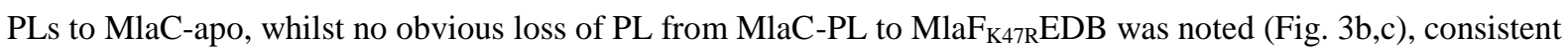
with our previous observations for MlaFEDB (Hughes et al. 2019). Loading of MlaC-apo with PLs directly from the liposome was discounted as we have previously shown this does not occur (Hughes et al. 2019). However, regardless of its ability to transport PLs to MlaC, $\mathrm{MlaF}_{\mathrm{K} 47 \mathrm{R}} \mathrm{EDB}$ lacked flippase activity (Fig. 3d \& Supplementary Fig. 5). This is in line with its ATPase inactivity; with the levels of inner leaflet NBD-PE remaining consistent $(47 \%)$ in both the presence and absence of ATP.

Together these results demonstrate the necessity of ATP hydrolysis for MlaFEDB driven flippase activity and highlight how MlaFEDB mediates two distinct functions, PL transfer to MlaC and lipid translocation between the leaflets of the IM.

\section{MlaFEDB is able to flip all major species of E.coli phospholipid}

To address whether MlaFEDB shows flippase preference for specific PL species present within the bacterial IM, we probed MlaFEDB flippase activity in the presence of headgroup NBD labelled phosphatidylglycerol (MlaFEDB:NBD-PG) and TopFluor® labelled cardiolipin (MlaFEDB:TopFluor®-CL), NBD headgroup labelled cardiolipin was not available. MlaFEDB displayed the ability to flip both phosphatidylglycerol and cardiolipin from the inner to the outer leaflet of the proteoliposome (Fig. 4 \& Supplementary Fig. 6a,b). Whilst the directionality of flippase activity remained constant for NBD-PE, NBD-PG, and TopFluor-CL, the levels at which flippase activity occurred differed between PL species. MlaFEDB was able to translocate NBD-PE and NBD-PG at similar rates in the presence of $1 \mathrm{mM}$ ATP, with levels of inner leaflet fluorescence decreasing to $\sim 18 \%$ for both species. However, the extent to which cardiolipin was translocated between leaflets by MlaFEDB was significantly reduced, with around $35 \%$ of Topfluor®-CL remaining in the inner leaflet following ATP incubation over the same time period (Fig. 4). Until now it has remained unclear whether the Mla pathway plays a role in cardiolipin transport, with Thong et al suggesting MlaC is unable to bind it (Thong et al. 2016), whilst we previously provided evidence for binding (Hughes et al. 2019). With the current observation of CL leaflet flipping by MlaFEDB, albeit at a lower level to PG and PE, it more firmly suggests the Mla pathway is able to transport CL. Whether the decreased levels of CL flipping are due to a preference for the diacyl chain containing PE and PG rather than the teta-acyl CL remains unclear however due to having to use an alternate CL fluorophore which could impact on rate. The results however demonstrate that MlaFEDB is able to function as a flippase for all three major PL species found within the bacterial membrane. Furthermore, the observation of flipping from the inner leaflet to the outer leaflet when presented with ATP on the exterior of the MlaFEDB proteoliposome corresponds to a bulk movement of PL towards the MlaF protein and hence within the cell envelope the movement of PLs from the outer to the inner leaflet of the IM.

MlaFEDB flippase activity correlates with MlaC phospholipid loading and transport towards the outer membrane. 
Following our observation that MlaFEDB mediates the flipping of PLs in a unidirectional fashion from the outer to the inner leaflet of the IM, a process which appears counterintuitive to our previous study showing MlaFEDB functions to extract PL from the IM and donates it to MlaC-apo and infers a shuttling of PLs towards the OM (Hughes et al. 2019), we sought to understand this process in more detail.

With the knowledge that $\sim 10 \%$ of the MlaFEDB complexes within the proteoliposome orient with the MlaD component outward facing (Fig. 1a,b), incubation of MlaFEDB:NBD-PE proteoliposomes with MlaC-apo yielded information regarding the leaflet from which PLs are exported to MlaC (Fig. 5a \& Supplementary Fig. 7). Upon incubation of MlaC-apo with MlaFEDB:NBD-PE proteoliposomes lacking ATP, a significant reduction in the inner leaflet level of NBD-PE was observed compared to the outer leaflet. As these assays were performed in the absence of ATP it can be inferred that this change in ratio was due to transfer to MlaC rather than flippase activity, certainly no flippase activity was previously noted in the absence of ATP. In contrast, the addition of MlaC-PL to MlaFEDB:NBD-PE fluorescently labelled proteoliposomes was seen to have no obvious effect on the levels of inner leaflet fluorescence. This being in line with the levels observed in untreated MlaFEDB:NBDPE proteoliposomes. These results therefore indicate that $\mathrm{MlaC}$ affects the levels of NBD-PE within the inner leaflet. MlaC mediated removal of PLs from the outer leaflet could not be measured using this method as the fluorescence signal for the outer leaflet was inferred from the total fluorescence minus inner leaflet fluorescence and therefore comprised of both MlaC-NBD-PE bound and outer leaflet NBD-PE.

To further investigate which bilayer leaflet PLs were extracted from we introduced NBD-PE into the outer leaflet of MlaFEDB E.coli polar lipid proteoliposomes (MlaFEDB:NBD-PE ${ }^{\text {OUTER}}$ ) using the approach of Chiantia et al. (Chiantia et al. 2012) and compared PL transfer capabilities to that of MlaFEDB proteoliposomes in which both leaflets were labelled (MlaFEDB:NBD-PE ${ }^{\text {BOTH }}$ ) (Supplementary Fig. 8). We could then incubate these proteoliposomes with MlaC-apo and through the use of TLC and fluorescence imaging monitor the transfer of NBD-PE (Fig. 5b). Following incubation with MlaFEDB:NBD-PE ${ }^{\text {BOTH }}$, as expected MlaC-apo became loaded with NBD-PE by the clear fluorescence observed on the TLC plate, thus indicating transfer of NBD-PE between MlaFEDB and MlaC. However, on incubation of MlaC-apo with MlaFEDB:NBD-PE ${ }^{\text {OUTER }}$ almost no fluorescence was observed. Density mapping estimated the signal to be approximately $10 \%$ of that following incubation with MlaFEDB:NBD-PE ${ }^{\text {Вотн }}$. PL transfer was still seen to occur as staining of the TLC plate with PMA highlighted MlaC-apo was loaded with PLs (Fig. 5c). This therefore suggests uptake occurred from the inner liposomal leaflet only. We attribute the $\sim 10 \%$ signal to the observation that IM labelling with NBD-PE wasn't $100 \%$. We observed $\sim 10 \%$ being incorporated into the inner leaflet thus some NBD-PE could gain access to MlaC. Incubation of MlaC-PL with MlaFEDB:NBD ${ }^{\text {OUTER }}$ or MlaFEDB:NBD ${ }^{\text {BOTH }}$ resulted in no change in observed fluorescence and an inability to donate or receive any PLs, suggesting the binding of PL to MlaC is sufficiently tight that exchange cannot occur.

These results therefore show that in the presence of MlaC-apo and in the absence of ATP, PLs are extracted from the inner leaflet of the MlaFEDB proteoliposome. In this scenario, where MlaC can only interact with the population of MlaFEDB with the MlaD component outward facing suggests that within the context of the cell that, in the absence of ATP, MlaC is loaded with PLs solely from the inner leaflet of the IM. 


\section{Discussion}

The mechanism of Mla driven PL transport remains a controversial subject, with conflicting views within the literature regarding the directionality of PL movement (Powers and Trent 2019). Recently we showed that in isolation MlaFEDB transfers PLs from MlaFEDB to MlaC and suggested that the Mla system might mediate the flux of PLs towards the OM (Hughes et al. 2019). However, the role of ATP within this process remained unclear, as we observed PL transport occurring without the need for an energy source. Despite this, whole cell studies performed by Kamishke and colleagues provided evidence to suggest that ATP is needed for Mla mediated transport of PLs toward the OM (Kamischke et al. 2019). As such, elucidating how ATP influences PL transport represents one of the key questions remaining regarding Mla mediated activity. Here we have attempted to reconcile these discrepancies and provide evidence to suggest MlaFEDB functions as a flippase in addition to its PL transport activity, and is able to flip PLs from the outer to the inner leaflet of the IM. We also show that on interaction with MlaFEDB and in the absence of ATP, MlaC gets loaded with PLs solely from the inner leaflet of the IM.

The significant MlaFEDB flippase activity observed in this study initially seemed at odds with our previous findings in which we observed an ATP independent export of PLs into the periplasm via MlaC (Hughes et al. 2019). Especially confusing was the observation of PL flipping towards the MlaF component, which in the context of the cell represents the movement of PLs from the outer to the inner leaflet, the opposite direction to PL export. These results in isolation provide credence to the idea that MlaFEDB could function in a retrograde transport fashion by redistributing PLs across both bilayers following influx from the OM. However, our previous observation showing PL export to MlaC (Hughes et al. 2019), the recently published structures of MlaFEDB adopting an exporter fold (Coudray et al. 2020, Mann et al. 2020), and our current findings casts doubt on this model. But how then can flippase activity and export be rationalised?

Work from our group, in addition to studies performed by Ekiert et al. (2017), have outlined the PL binding mechanism of MlaC (Ekiert et al. 2017, Hughes et al. 2019), with fatty acid chains buried deep within the MlaC binding pocket, whilst the head group protrudes from the protein surface. PLs must therefore be orientated on MlaD such that when MlaC binds the acyl tails are presented to it. Our observation that MlaC is loaded with inner leaflet PLs in the absence of ATP can therefore be rationalised as the PLs in this leaflet are already positioned in the correct orientation with their tails presented (Fig. 6) and thus represents both an energetically and spatially favourable mechanism of PL transfer. In this scenario, PLs could be directly exported to MlaC via a continuous flow driven by the affinity of MlaC for PLs and its prevalence. Further support for this model is given by the recently published structure of the A. Baumannii MlaFEDB complex in which a channel appears to be observed through MlaE from PLs bound to MlaFEDB on the inner leaflet side directly to MlaD (Mann et al. 2020). What then is the role of the ATPase driven flippase action? This at first glance is somewhat more difficult to reconcile. Is this just an aberrant effect due to the use of fluorescently labelled PLs? Or if real, why is there a need for PLs to be flipped when they can be extracted directly from the inner leaflet without ATP hydrolysis? We propose it is real and is backed up by the recently released structure of E. coli MlaFEDB (Coudray et al. 2020). This complex was solved in the apo configuration with PLs observed within the core of the MlaE dimer. Although presented as the "outward-open" configuration, neither PL was observed in the tail up configuration as would be expected for export to MlaD and more likely represents the MLaFEDB complex prior to flipping of PLs to the inner leaflet. What then is the role of the flippase? We propose it is there to enable PLs to be extracted from both leaflets, thus 
maintaining membrane homeostasis. If extraction only occurred from the inner leaflet, curvature would ensue (McMahon and Gallop 2005, Zimmerberg and Kozlov 2006, Devaux et al. 2008). Flippase activity would therefore enable transfer between bilayers and would thus allow extraction from both leaflets, the assumption being that two PLs are exported from the inner leaflet for every one flipped from the outer leaflet, resulting in a net loss of one PL from each leaflet. Within the cell ATP levels are sufficiently high that this process would likely be continual but presumably the export and flippase activity of MlaFEDB must be coordinated. However, in our in vitro study the observation of flippase activity and export apparently occurring independently seems somewhat at odds with this, and in truth we currently can't provide a clear answer for this observation but it must be noted we have previously shown that ATPase activity is upregulated in the presence of MlaC-apo implying coordination between the two processes does occur. It must also be noted that MlaB is a STAS domain containing protein. STAS domains have previously been implicated in regulation (Sharma et al. 2011), therefore it is feasible to suggest that the STAS domain containing MlaB might also participate in this. Indeed, Thong et al. (2016) have previously outlined a role for MlaB in influencing MlaFEDB ATPase activity (Thong et al. 2016), a notion recently reinforced by Kolich et al. (Kolich et al. 2020), however the exact mechanism of this regulation remains to be elucidated. As such it could be that some form of dysregulation by MlaB has occurred in our in vitro experiments that led to the disconnect between flippase activity and export. Indeed Mann et al (2020) noted only $50 \%$ of their cryo-EM class averages having MlaB bound to both MlaF proteins, the remaining only had one copy (Mann et al. 2020). It is therefore not inconceivable to assume the same could happen here.

In summary, this study provides evidence to suggest that MlaFEDB exhibits PL flippase activity, and can facilitate the transbilayer movement of phosphatidylethanolamine, phosphatidylglycerol and cardiolipin from the outer to the inner leaflet of the IM. We also show that in the absence of ATP MlaFEDB mediates the transfer of PLs from the inner leaflet of the IM to MlaC within the periplasm. These findings highlight MlaFEDB as the first generic PL flippase discovered in Gram-negative bacteria and provide further mechanistic details to support the Mla driven anterograde PL transport process.

\section{Materials and Methods}

\section{Expression and purification of MlaFEDB}

MlaFEDB was expressed and purified following methodology as described by (Ekiert et al. 2017). Briefly, Rosetta 2 (DE3) cells (Novagen) were transformed with the plasmid pBE1196 (complete mlaFEDCB operon containing a hexa-histidine tag at the $\mathrm{C}$ terminus of $\mathrm{MlaF}$ ). $\mathrm{MlaF}_{\mathrm{K} 47 \mathrm{R}} \mathrm{EDB}$ mutants were generated through site-directed mutagenesis using the MlaFEDB pBE1196 plasmid as target DNA and purified via the same protocol as MlaFEDB. Firstly, overnight cultures were grown to an OD600 of 0.9 with shaking at $37^{\circ} \mathrm{C}$. Addition of arabinose to a final concentration of $0.1 \%(\mathrm{w} / \mathrm{v})$ with 4 hours shaking incubation at $37{ }^{\circ} \mathrm{C}$ was used to induce MlaFEDB expression. The cells were pelleted through centrifugation at $6,000 \mathrm{~g}$ for 10 mins before resuspension in $50 \mathrm{mM}$ Tris $\mathrm{pH} 8,150 \mathrm{mM} \mathrm{NaCl}, 10 \mathrm{mM}$ imidazole. The cells were then lysed through three passes through an Emulsiflex C3 disruptor (Avestin). Lysate was then subjected to two rounds of centrifugation, the first $(20,000 \mathrm{~g}, 30 \mathrm{~min})$ to remove the cell debris, and the second $(100,000 \mathrm{~g}, 30 \mathrm{~min})$ to pellet membranes. The membrane pellets were solubilized for $1 \mathrm{~h}$ at $4{ }^{\circ} \mathrm{C}$ in $50 \mathrm{mM}$ Tris $\mathrm{pH} 8,500 \mathrm{mM} \mathrm{NaCl}, 10 \mathrm{mM}$ imidazole, $25 \mathrm{mM}$ DDM, before insoluble 
material was removed by centrifugation at $100,000 \mathrm{~g}$ for $30 \mathrm{mins}$. Solubilized membranes were then bound to a 5ml Ni-NTA column (HisTrap, GE Healthcare), which was subsequently washed with $50 \mathrm{mM}$ Tris pH 8, $150 \mathrm{mM}$ $\mathrm{NaCl}, 50 \mathrm{mM}$ imidazole, $0.5 \mathrm{mM}$ DDM, before bound protein was eluted with $50 \mathrm{mM}$ Tris $\mathrm{pH} 8,150 \mathrm{mM} \mathrm{NaCl}$, $250 \mathrm{mM}$ imidazole, $0.5 \mathrm{mM}$ DDM. MlaFEDB containing fractions were then subjected to additional purification on a Superdex 200 size-exclusion column (GE Healthcare) equilibrated in $50 \mathrm{mM}$ Tris pH 8, $150 \mathrm{mM} \mathrm{NaCl}, 0.5$ mM DDM.

\section{Expression and purification of MlaC}

A custom plasmid (pBE1203) containing DNA corresponding to MlaC with an N-terminal hexa-histidine tag followed by a TEV protease cleavage site (Ekiert et al. 2017) was transformed into E.coli BL21(DE3) cells. Overnight cultures were grown in lysogeny broth at $37^{\circ} \mathrm{C}$ to an $\mathrm{OD} 600=0.6$ whereupon protein expression was induced by the addition of isopropyl- $\beta$-d-thiogalactoside to a final concentration of $1 \mathrm{mM}$, cells were then continually grown overnight at $15{ }^{\circ} \mathrm{C}$. The cells pelleted through centrifugation at $6,000 \mathrm{~g}$ for 10 mins and then resuspended in $50 \mathrm{mM}$ Tris $\mathrm{pH} 8,500 \mathrm{mM} \mathrm{NaCl}, 10 \mathrm{mM}$ imidazole. Cell pellets were lysed by three passes through an Emulsiflex C3 disruptor (Avestin) and then centrifuged (75,000g, $45 \mathrm{~min}$ ) to remove cell debris. Clarified lysate was then flowed over a 5-ml Ni-NTA column (GE Healthcare), before being washed with $50 \mathrm{mM}$

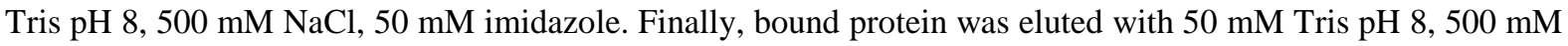
$\mathrm{NaCl}, 500 \mathrm{mM}$ imidazole. MlaC containing fractions were further purified through size exclusion chromatography

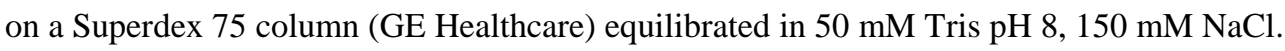

\section{Lipid removal from MlaC}

Generating MlaC-Apo was performed as described previously by (Hughes et al. 2019). Briefly, purified MlaC was bound to a Ni-NTA affinity column (GE Healthcare) and washed with $25 \mathrm{ml}$ of $50 \mathrm{mM}$ Tris $\mathrm{pH} 8,500 \mathrm{mM}$ $\mathrm{NaCl}, 10 \mathrm{mM}$ imidazole, $25 \mathrm{mM} \beta-\mathrm{OG}$ followed by a $1 \mathrm{~h}$ static incubation period in the wash buffer. This process of washing and incubation was repeated 3 times before being followed by a final wash with $50 \mathrm{ml}$ of $50 \mathrm{mM}$ Tris $\mathrm{pH} 8,500 \mathrm{mM} \mathrm{NaCl}, 10 \mathrm{mM}$ imidazole. MlaC was then eluted using $50 \mathrm{mM}$ Tris $\mathrm{pH} 8,500 \mathrm{mM} \mathrm{NaCl}, 250 \mathrm{mM}$ imidazole and dialysed against $50 \mathrm{mM}$ Tris $\mathrm{pH} 8,150 \mathrm{mM} \mathrm{NaCl}$.

\section{Formation of proteoliposomes}

Proteoliposomal formation was based on methodologies performed by (Eckford and Sharom 2010). Briefly, either solely $10 \mathrm{mg}$ of E.coli polar lipids (Avanti Polar Lipids), or doped with either $0.3 \%$ (w/w) NBD-PE, $0.3 \%$ (w/w) NBD-PG, or $0.3 \%(\mathrm{w} / \mathrm{w})$ TopFluorCL were dried down under a constant stream of nitrogen. Dried lipid samples were partially solubilized in $250 \mu \mathrm{L}$ in $50 \mathrm{mM}$ Tris $\mathrm{pH} 8,150 \mathrm{mM} \mathrm{NaCl}, 0.5 \mathrm{mM}$ DDM before $1 \mathrm{mg}$ of purified protein in DDM (MlaFEDB, MlaF $\left.\mathrm{K}_{47 \mathrm{R}} \mathrm{EDB}\right)$ was added, producing a final lipid:protein ratio of 10:1 (w/w). Samples were then incubated on ice for 30 mins with occasional mixing before being separated on a Superdex 75 gel filtration column equilibrated in $50 \mathrm{mM}$ Tris $\mathrm{pH} 8,150 \mathrm{mM} \mathrm{NaCl}, 5 \mathrm{mM} \mathrm{MgCl}$. Proteoliposomes eluted in the column void volume, were pooled and adjusted to give a final protein concentration of $0.5 \mathrm{mg} / \mathrm{ml}$.

To solely label the outer leaflet of MlaFEDB proteoliposomes with NBD-PE a method outlined in (Chiantia et al. 2012) was adapted. Briefly, NBD-PE lipids were dried down and resuspended in $100 \%$ Ethanol to produce a stock concentration of $20 \mathrm{mg} / \mathrm{ml}$. These lipids were then added to formed MlaFEDB proteoliposomes 
as outlined above to produce a final NBD-PE concentration of $0.3 \%(\mathrm{w} / \mathrm{w})$. Proteoliposomes were subsequently separated from non-inserted free NBD-PE molecules through separation on a Superdex 75 gel filtration column equilibrated in $50 \mathrm{mM}$ Tris $\mathrm{pH} 8,150 \mathrm{mM} \mathrm{NaCl}, 5 \mathrm{mM} \mathrm{MgCl} 2$.

In those experiments in which proteoliposomes contained differential leaflet labelling, distinction has been made in which proteoliposomes containing NBD-PE in the outer leaflet alone are termed MlaFEDB:NBDPE $E^{\text {OUTER }}$ whilst those proteoliposomes labelled with NBD-PE in both inner and outer leaflets are termed MlaFEDB:NBD-PE ${ }^{\text {BOTH }}$.

\section{Lipid translocation / flippase assay}

The flippase activity of various MlaFEDB constructs was determined through measuring differences in the ratios of NBD labelled lipids across the different leaflets of the proteoliposome. Flippase assays were performed using the same methodology regardless of which liposomal/proteoliposomal system was used. Firstly, proteoliposomes were subjected to a variety of different conditions, including incubation with a range of ATP concentrations $(0$ $1 \mathrm{mM}$ ), $1 \mathrm{mM} \mathrm{ATP} \gamma \mathrm{S}, 1 \mathrm{mM}$ GTP, or either $0.5 \mathrm{mg} / \mathrm{ml}$ MlaC-Apo or MlaC-PL. Incubation was performed over an hour period at room temperature, and for flippase specific assays in the presence of an ATP regeneration system (0.5 mM Phosphoenolpyruvate (Sigma-Aldrich) and $100 \mathrm{U} \mathrm{ml-1}$ pyruvate kinase (Sigma-Aldrich)). Following incubation, the reaction mixture was transferred to a 96-well fluorescence based assay microplate (Thermo Fisher Scientific) (200 $\mu \mathrm{L}$ reaction volume) and fluorescence emission (NBD-Lipids: excitation at $464 \mathrm{~nm}$, emission at $536 \mathrm{~nm}$; TopFluor-CL: excitation at $498 \mathrm{~nm}$, emission at $507 \mathrm{~nm}$ ) monitored at $25^{\circ} \mathrm{C}$ until a stable baseline was established. $4 \mathrm{mM}$ Sodium Dithionite was added to the reaction mixture and fluorescence again monitored until readings stabilized. Finally, $1 \%$ (w/v) Triton X-100 was added, with fluorescence measured to give a background reading.

\section{Dynamic light scattering}

Dynamic light scattering experiments were performed using a DynaPro® Plate Reader III and DYNAMICS software (Wyatt Technology, Haverhill, UK), using the laser wavelength of $825.4 \mathrm{~nm}$ with a detector angle of $150^{\circ}$. Each sample $(40 \mu \mathrm{L})$ was loaded into a 384 -well glass bottom SensoPlate ${ }^{\mathrm{TM}}$ (Greiner Bio-One, Germany) in triplicate. Each measurement consisted of $10 \mathrm{scans}$ of $5 \mathrm{~s}$, carried out at $25^{\circ} \mathrm{C}$, with the attenuator position and laser power automatically optimized for size determination $(\mathrm{nm})$.

\section{ATPase assay}

An NADH enzyme-linked assay (Norby 1988) that was adapted for a microplate reader (Kiianitsa et al. 2003) was used to determine either the MlaFEDB or $\mathrm{MlaF}_{\mathrm{K} 47 \mathrm{R}} \mathrm{EDB}$ rate of ATP hydrolysis. $0.1 \mu \mathrm{M}$ of protein, $200 \mathrm{mM}$ NADH (Sigma-Aldrich), $20 \mathrm{U} \mathrm{ml-1} \mathrm{lactic} \mathrm{dehydrogenase} \mathrm{(Sigma-Aldrich),} 100 \mathrm{U} \mathrm{ml}^{-1}$ pyruvate kinase (SigmaAldrich), 0.5 mM phosphoenolpyruvate (Sigma-Aldrich) and different ATP (Sigma-Aldrich) concentrations were added to the wells of a 96-well plate (Sigma-Aldrich) and made up in assay buffer (50 mM Tris pH 8.0, $150 \mathrm{mM}$ $\mathrm{NaCl}, 5 \mathrm{mM} \mathrm{MgCl} 2,0.5 \mathrm{mM} \mathrm{DDM})$ to produce a total final assay volume of $75 \mu$ l. Using an Anthos Zenyth 340rt (Biochrom) absorbance photometer equipped with ADAP software the change in absorbance at $340 \mathrm{~nm}$ was measured. Reactions were followed for 10-60 min with measurements taken every 15s. Using maximal $340 \mathrm{~nm}$ absorbance readings (with $0 \mu \mathrm{M}$ ATP) and minimal $340 \mathrm{~nm}$ absorbance readings (with $0 \mu \mathrm{M}$ NADH) ATP- 
hydrolysis rates were determined; the concentration of NADH at each time-point was calculated from these readings, allowing for a linear fit of the reduction in NADH absorbance and conversion to ATP hydrolysis.

\section{Thin layer chromatography}

PL movement within the Mla system was monitored through a process of incubation between different MlaFEDB proteoliposomes and $\mathrm{MlaC}$ in either its -Apo or -PL forms followed by separation through SEC. Proteoliposomes including MlaFEDB:PL, MlaF ${ }_{\mathrm{K} 47 \mathrm{R}}$ EDB:NBD-PE, MlaFEDB:NBD-PE ${ }^{\text {BOTH }}$, and MlaFEDB:NBD-PE ${ }^{\text {OUTER }}$ were adjusted to $2 \mathrm{mg} / \mathrm{ml}$ and incubated in solution with either MlaC-Apo or MlaC-PL at $2 \mathrm{mg} / \mathrm{ml}$ for 1 hour. Following incubation, proteoliposomes were separated from MlaC through SEC using a Superdex 75 column (GE Healthcare) equilibrated in $50 \mathrm{mM}$ Tris $\mathrm{pH} 8,150 \mathrm{mM} \mathrm{NaCl}$. Samples were then collected for lipid extraction.

$2 \mathrm{ml}$ of sample was adjusted to $0.5 \mathrm{mg} / \mathrm{ml}$ and added to $2 \mathrm{ml}$ methanol and $1 \mathrm{ml}$ chloroform. Samples were then vortexed for $5 \mathrm{~min}$, incubated for $30 \mathrm{~min}$ at $50{ }^{\circ} \mathrm{C}$ and vortexed again for $5 \mathrm{~min}$. The mixture was centrifuged $(2,000 \mathrm{~g}, 10 \mathrm{~min})$, and the lower phase was extracted and evaporated. Dried lipids were resuspended in $100 \mu \mathrm{l}$ chloroform, and $5 \mu \mathrm{l}$ was loaded onto a Silica TLC plate (Sigma) and run with a 6.5:2.5:1 (chloroform:methanol:acetic acid) solvent. The TLC plate was dried for $30 \mathrm{~min}$, stained with 10\% (w/v) phosphomolybdic acid in ethanol and heated until staining occurred. For detection of NBD-lipid fluorescence Silica TLC plates were left unstained and visualised using an Amersham Imager 680 blot and gel imager (GE Healthcare).

\section{Western blotting}

In order to determine the orientation of MlaFEDB once reconstituted into polar lipid liposomes, MlaFEDB:NBDPE was subjected to Proteinase K digestion. $1 \mathrm{mg}$ of MlaFEDB:NBD-PE was incubated with $0.05 \mathrm{mg} / \mathrm{ml}$ Proteinase K (Thermo Fisher) for $10 \mathrm{mins}$ at $4{ }^{\circ} \mathrm{C}$. In order to ensure the stability of the proteoliposomes, a control sample of MlaFEDB:NBD-PE was treated with $1 \%$ (v/v) Triton X-100 before being subjected to Proteinase K digestion as outlined above. Proteinase K digested proteoliposomes in both unsolubilzed and Triton X-100 solubilized conditions were compared to samples that were left undigested. All samples were then boiled at $95{ }^{\circ} \mathrm{C}$ for 10 mins at a 1:1 (v/v) ratio in 2x Laemmli loading dye (Sigma) before being electrophoresed through a 4-12 $\%$ NuPAGE Bis-Tris polyacrylamide gel (BioRad). Protein bands were then transferred from the gels onto 0.2 $\mu \mathrm{m}$ polyvinylidene fluoride (PVDF) membranes (Bio-Rad, CA, USA) using semi-dry electroblotting system (Trans-Blot Turbo Transfer System, Bio-Rad). Membranes were blocked for 1 hour with 5\% (w/v) dried milk powder dissolved in $10 \mathrm{mM}$ Tris pH 8, $150 \mathrm{mM} \mathrm{NaCl}, 0.1 \%$ (v/v) Tween-20 (TBST). Membranes were then incubated for 4 hours with anti-His hexahistidine antisera conjugated to horseradish peroxidase (HRP) (Sigma) diluted in TBST at a ratio of 1:5000. Following 3 washes in TBST, membranes were incubated with Rabbit AntiMouse IgG secondary antisera (Abcam) diluted at a ratio of 1:25000 in TBST. An enhanced chemiluminescence (ECL) detection kit (Merck Millipore) was used to develop the membranes, with chemiluminescence signals visualized using a cp1000 film processor (Agfa).

\section{Acknowledgements}


bioRxiv preprint doi: https://doi org/10.1101/2020.06.06.138008; this version posted June 6, 2020. The copyright holder for this preprint (which was not certified by peer review) is the author/funder, who has granted bioRxiv a license to display the preprint in perpetuity. It is made available under aCC-BY-NC-ND 4.0 International license.

We thank D. Ekiert and G. Bhabha for discussion and kindly providing Mla plasmid constructs. This research was supported by BBSRC grants BB/P009840/1 and BB/S017283/1 (GWH, PS, \& TJK) and BBSRC PhD funding through the Midlands Integrative Biosciences Training Partnership (PJW, SAN, \& BFC).

\section{Author Contributions}

GWH, PS, SAN, PJW, BFC and TJK all participated in the conception and design of the work. GWH, PS, SAN, PJW, BFC and TJK all participated in the data acquisition, analysis or interpretation of the work. GWH, PJW, BFC and TJK were involved in writing and editing the manuscript. All authors approved the final version submitted for publication.

\section{Competing Interests}

The authors declare that they have no competing interests with the contents of this article.

\section{References}

Abellon-Ruiz, J., S. S. Kaptan, A. Basle, B. Claudi, D. Bumann, U. Kleinekathofer and B. van den Berg (2017). "Structural basis for maintenance of bacterial outer membrane lipid asymmetry." Nat Microbiol 2(12): 1616-1623.

Chiantia, S., A. S. Klymchenko and E. London (2012). "A novel leaflet-selective fluorescence labeling technique reveals differences between inner and outer leaflets at high bilayer curvature." Biochim Biophys Acta 1818(5): 1284-1290.

Chng, S. S., L. S. Gronenberg and D. Kahne (2010). "Proteins required for lipopolysaccharide assembly in Escherichia coli form a transenvelope complex." Biochemistry 49(22): 4565-4567.

Chong, Z. S., W. F. Woo and S. S. Chng (2015). "Osmoporin OmpC forms a complex with MlaA to maintain outer membrane lipid asymmetry in Escherichia coli." Mol Microbiol 98(6): 1133-1146.

Coudray, N., G. L. Isom, M. R. Macrae, M. N. Saidddin, G. Bhabha and D. C. Ekiert (2020). "Structure of MlaFEDB lipid transporter reveals an ABC exporter fold and two bound phospholipids." BioRxiv.

Devaux, P. F., A. Herrmann, N. Ohlwein and M. M. Kozlov (2008). "How lipid flippases can modulate membrane structure." Biochim Biophys Acta 1778(7-8): 1591-1600.

Eckford, P. D. and F. J. Sharom (2005). "The reconstituted P-glycoprotein multidrug transporter is a flippase for glucosylceramide and other simple glycosphingolipids." Biochem J 389(Pt 2): 517-526.

Eckford, P. D. and F. J. Sharom (2010). "The reconstituted Escherichia coli MsbA protein displays lipid flippase activity." Biochem J 429(1): 195-203. 
bioRxiv preprint doi: https://doi org/101101/2020.06.06.138008; this version posted June 6 , 2020. The copyright holder for this preprin (which was not certified by peer review) is the author/funder, who has granted bioRxiv a license to display the preprint in perpetuity. It is made available under aCC-BY-NC-ND 4.0 International license.

Ekiert, D. C., G. Bhabha, G. L. Isom, G. Greenan, S. Ovchinnikov, I. R. Henderson, J. S. Cox and R. D. Vale (2017). "Architectures of Lipid Transport Systems for the Bacterial Outer Membrane." Cell 169(2): 273-285 e217.

Ercan, B., W. Y. Low, X. Liu and S. S. Chng (2018). "Characterization of Interactions and Phospholipid Transfer between Substrate Binding Proteins of the OmpC-Mla System." Biochemistry.

Hughes, G. W., S. C. L. Hall, C. S. Laxton, P. Sridhar, A. H. Mahadi, C. Hatton, T. J. Piggot, P. J. Wotherspoon, A. C. Leney, D. G. Ward, M. Jamshad, V. Spana, I. T. Cadby, C. Harding, G. L. Isom, J. A. Bryant, R. J. Parr, Y. Yakub, M. Jeeves, D. Huber, I. R. Henderson, L. A. Clifton, A. L. Lovering and T. J. Knowles (2019). "Evidence for phospholipid export from the bacterial inner membrane by the Mla ABC transport system." Nat Microbiol. 4(10): 1692-1705

Kamio, Y. and H. Nikaido (1976). "Outer membrane of Salmonella typhimurium: accessibility of phospholipid head groups to phospholipase $\mathrm{c}$ and cyanogen bromide activated dextran in the external medium." Biochemistry 15(12): 2561-2570.

Kamischke, C., J. Fan, J. Bergeron, H. D. Kulasekara, Z. D. Dalebroux, A. Burrell, J. M. Kollman and S. I. Miller (2019). "The Acinetobacter baumannii Mla system and glycerophospholipid transport to the outer membrane." Elife 8.

Kiianitsa, K., J. A. Solinger and W. D. Heyer (2003). "NADH-coupled microplate photometric assay for kinetic studies of ATP-hydrolyzing enzymes with low and high specific activities." Anal Biochem 321(2): 266-271.

Kolich, L., Y.-T. Chang, N. Coudray, S. I. Giacometti, M. R. Macrae, G. L. Isom, E. M. Teran, G. Bhabha and D. C. Ekiert (2020). "Structure of MlaFB uncovers novel mechanisms of ABC transporter regulation." BioRxiv.

Malinverni, J. C. and T. J. Silhavy (2009). "An ABC transport system that maintains lipid asymmetry in the gram-negative outer membrane." Proc Natl Acad Sci U S A 106(19): 8009-8014. Mann, D., J. Fan, D. P. Farrell, K. Somboon, A. Muenks, S. B. Tzokov, S. Khalid, F. Dimaio, S. I. Miller and J. R. C. Bergeron (2020). "Structural basis for lipid transport 1 by the MLA complex." BioRxiv.

Marrink, S. J., O. Berger, P. Tieleman and F. Jahnig (1998). "Adhesion forces of lipids in a phospholipid membrane studied by molecular dynamics simulations." Biophys J 74(2 Pt 1): 931-943. McMahon, H. T. and J. L. Gallop (2005). "Membrane curvature and mechanisms of dynamic cell membrane remodelling." Nature 438(7068): 590-596.

Nikaido, H. (2005). "Restoring permeability barrier function to outer membrane." Chem Biol 12(5): 507-509.

Norby, J. G. (1988). "Coupled assay of Na+,K+-ATPase activity." Methods Enzymol 156: 116-119.

Okuda, S., D. J. Sherman, T. J. Silhavy, N. Ruiz and D. Kahne (2016). "Lipopolysaccharide transport and assembly at the outer membrane: the PEZ model." Nat Rev Microbiol 14(6): 337-345.

Powers, M. J. and M. S. Trent (2019). "Intermembrane transport: Glycerophospholipid homeostasis of the Gram-negative cell envelope." Proc Natl Acad Sci U S A 116(35): 17147-17155.

Roier, S., F. G. Zingl, F. Cakar, S. Durakovic, P. Kohl, T. O. Eichmann, L. Klug, B. Gadermaier, K. Weinzerl, R. Prassl, A. Lass, G. Daum, J. Reidl, M. F. Feldman and S. Schild (2016). "A novel mechanism for the biogenesis of outer membrane vesicles in Gram-negative bacteria." Nat Commun 7: 10515 . 
bioRxiv preprint doi: https://doi org/101101/2020.06.06.138008; this version posted June 6, 2020. The copyright holder for this preprint (which was not certified by peer review) is the author/funder, who has granted bioRxiv a license to display the preprint in perpetuity. It is made available under aCC-BY-NC-ND 4.0 International license.

Ruiz, N., D. Kahne and T. J. Silhavy (2006). "Advances in understanding bacterial outer-membrane biogenesis." Nat Rev Microbiol 4(1): 57-66.

Sharma, A. K., A. C. Rigby and S. L. Alper (2011). "STAS domain structure and function." Cell Physiol Biochem 28(3): 407-422.

Sutterlin, H. A., H. Shi, K. L. May, A. Miguel, S. Khare, K. C. Huang and T. J. Silhavy (2016). "Disruption of lipid homeostasis in the Gram-negative cell envelope activates a novel cell death pathway." Proc Natl Acad Sci U S A 113(11): E1565-1574.

Thong, S., B. Ercan, F. Torta, Z. Y. Fong, H. Y. Wong, M. R. Wenk and S. S. Chng (2016). "Defining key roles for auxiliary proteins in an $\mathrm{ABC}$ transporter that maintains bacterial outer membrane lipid asymmetry." Elife $\mathbf{5}$.

Tran, A. X., M. S. Trent and C. Whitfield (2008). "The LptA protein of Escherichia coli is a periplasmic lipid A-binding protein involved in the lipopolysaccharide export pathway." J Biol Chem 283(29): 20342-20349.

Zimmerberg, J. and M. M. Kozlov (2006). "How proteins produce cellular membrane curvature." Nat Rev Mol Cell Biol 7(1): 9-19.

\section{Figure Legends}

Figure 1. MlaFEDB orientates differentially in liposomes. A) MlaFEDB:NBD-PE was treated with proteinase $\mathrm{K}$ and western blotting performed, staining with an anti-His antibody, allowing for identification of MlaF. Owing to the impermeability of the MlaFEDB:NBD-PE to proteinase $\mathrm{K}$ the orientation of the MlaFEDB complex within the liposome could then be assessed, with any observed anti-His staining following proteinase $\mathrm{K}$ treatment resulting from inward facing MlaF. Western blots were then analysed through densitometry to allow quantification of protein orientation. B) Schematic diagram showing the preferential orientation of MlaFEDB within liposomes.

Figure 2. MlaFEDB has phospholipid flippase activity. A) MlaFEDB:NBD-PE proteoliposomes were incubated in the presence or absence of $1 \mathrm{mM}$ ATP and a regenerating system for 1 hour. NBD-PE fluorescence was then monitored. Proteoliposomes were then incubated with $4 \mathrm{mM}$ Sodium dithionite (first interruption in trace), with subsequent fluorescence levels observed being representative of inner leaflet NBD-PE levels. Background fluorescence levels were then established through the addition of 1\% (w/v) Triton X-100. In the presence of ATP levels of inner leaflet NBD-PE were substantially reduced as compared to untreated proteoliposomes, indicating flippase activity. B) The flippase assay as described above was performed using PL:NBD-PE liposomes. ATP did not influence the distribution of NBD-PE across the leaflets of the liposome. C) MlaFEDB:NBD-PE flippase assays were performed in the presence of a range of ATP concentrations from 0 - 1 $\mathrm{mM}$.

Figure 3. MlaFEDB flippase activity is dependent upon ATP hydrolysis. A) MlaFEDB:NBD-PE proteoliposomes were incubated for an hour in the presence of $1 \mathrm{mM} \mathrm{ATP,} 1 \mathrm{mM} \mathrm{ATP} \gamma \mathrm{S}, 1 \mathrm{mM} \mathrm{GTP}$, or left 
untreated. Following incubation, the levels of NBD-PE distributed across the leaflets of the proteoliposome were determined through dithionite quenching, allowing us to assess flippase activity. Bars represent the average results from 3 independent flippase assays, with standard deviation shown. $* * * * \mathrm{P}<0.0001$ as determined through

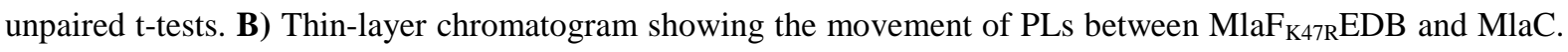
C) SDS-PAGE of the samples shown in $\mathbf{B}$ confirming separation of the various species following incubation. D) $\mathrm{MlaF}_{\mathrm{K} 4 \mathrm{R}} \mathrm{EDB}$ reconstituted into E.coli polar lipid liposomes containing $0.3 \%$ (w/w) NBD-PE were incubated either in the presence or absence of ATP and monitored for flippase activity through the use of dithionite quenching. Levels of inner leaflet NBD-PE were quantified for 3 individual assays, error bars represent standard deviation. Ns = no significance.

Figure 4. MlaFEDB mediated flippase activity occurs in the presence of all major E.coli phospholipids. E.coli polar lipid proteoliposomes containing reconstituted MlaFEDB and either $0.3 \%$ (w/w) NBD-PE, $0.3 \%$ (w/w) NBD-PG, or $0.3 \%$ (w/w) TopFluorCL were incubated for an hour in the presence or absence of $1 \mathrm{mM}$ ATP and a regenerating system. The levels of inner leaflet fluorescent lipid was determined through dithionite quenching. All datasets represent the average results from 3 independent flippase assays, with standard deviation shown. Ns: no significance, $* * \mathrm{P}<0.01, * * * \mathrm{P}<0.001, * * * * \mathrm{P}<0.0001$ as determined through unpaired t-tests.

Figure 5. MlaFEDB flippase activity facilitates PL transfer onto MlaC. A) MlaFEDB:NBD-PE proteoliposomes were incubated for an hour at a 1:1 molar ratio with either MlaC-Apo, MlaC-PL, or left untreated. The transbilayer distribution of NBD-PE following this incubation was determined through the use of dithionite quenching. Only in the presence of MlaC-Apo was inner leaflet fluorescence decreased. Bars represent the average results from 3 independent flippase assays, with standard deviation shown. Ns: no significance, $* * * * \mathrm{P}<0.0001$ as determined through unpaired t-tests. B) To further assess the ability of MlaC to accept PLs from the inner leaflet of MlaFEDB proteoliposomes we reconstituted MlaFEDB into E.coli polar lipid liposomes in which only

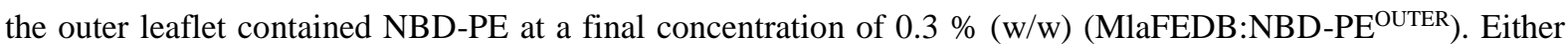
MlaFEDB:NBD-PE ${ }^{\text {OUTER }}$ or MlaFEDB reconstituted into polar lipid liposomes containing $0.3 \%$ (w/w) NBD-PE distributed across both leaflets (MlaFEDB:NBD-PE ${ }^{\text {BOTH }}$ ) were incubated with either MlaC-Apo or MlaC-PL. The transfer of NBD-PE between these species was then assessed through fluorescence visualization after separation via TLC. Labels indicate what protein lipids have been extracted from, following incubations with other proteins denoted by brackets. C) Identical TLC as shown in b following staining with $10 \%$ (w/v) phosphomolybdic acid (PMA) labelling mimics that of $\mathbf{b}$.

\section{Figure 6. Schematic diagram highlighting how MlaFEDB flippase activity correlates with PL transport} onto MlaC. Within the context of the cell, MlaFEDB flippase activity mediates the translocation of PLs from the outer to the inner leaflet of the IM. We suggest that this flippase activity is important in maintaining the integrity of the IM. We suggest that MlaC is loaded solely with inner leaflet PLs via MlaD, as such MlaFEDB flippase activity is required to replenish the lipids in the inner leaflet that are lost upon transfer to MlaC. In light of new structural evidence regarding MlaFEDB, we suggest flippase activity plays an essential role in orientating PLs to allow for the effective transfer onto MlaC. ATP hydrolysis may allow for outer leaflet PLs present within the complex to translocate its inner leaflet side, the subsequent change in conformation may then also allow for an 
extra inner leaflet PL to access the MlaE cavity. This would create a situation in which two PLs are poised for movement through the MlaE central cavity and loading onto MlaD. This PL movement through the cavity may rely on the action of MlaC stripping PLs previously bound to MlaD, which in turn increases MlaFEDB ATP hydrolysis, thus facilitating increased PL loading onto MlaD.

Supplementary Figure 1. MlaFEDB reconstitution into liposomes. A) Size-exclusion chromatogram and SDSPAGE showing the reconstitution of MlaFEDB into proteoliposomes consisting of E.coli polar lipids containing $0.3 \%(w / w)$ NBD-PE (MlaFEDB:NBD-PE). B) DLS measurement showing size distribution of MlaFEDB:NBDPE proteoliposomes.

Supplementary Figure 2. MlaFEDB flippase activity increases in an ATP concentration dependent fashion. Kinetic fluorescence plot displaying the effect of dithionite quenching on the flippase activity assay corresponding to Fig. 2c.

Supplementary Figure 3. The flippase activity of MlaFEDB relies on ATP hydrolysis. Flippase assay plot corresponding to Fig. 3a displaying full kinetic fluorescence data.

Supplementary Figure 4. MlaFk47REDB is unable to hydrolyse ATP. A) Size-exclusion chromatogram and corresponding SDS-PAGE of detergent-solubilized $\mathrm{MlaF}_{\mathrm{K} 47 \mathrm{R}} \mathrm{EDB}$ yielding a single complex. B) Enzyme coupled ATPase assay of $\mathrm{MlaF}_{\mathrm{K} 47 \mathrm{R}} \mathrm{EDB}(0.1 \mu \mathrm{M})$ compared to MlaFEDB $(0.1 \mu \mathrm{M})$ performed in detergent micelles $(0.05 \% \mathrm{DDM})$. The average ATP-hydrolysis rates were plotted against the ATP concentrations and fitted against an expanded Michaelis-Menten equation that includes a term for the Hill coefficient (n). For MlaFEDB, Vmax = $0.05 \mu \mathrm{M}$ ATP $\mathrm{s}-1, \mathrm{kcat}=0.5 \pm 0.1 \mathrm{~s}-1, \mathrm{~K}_{\mathrm{m}}=200.0 \pm 44.6 \mu \mathrm{M}$ and $\mathrm{n}=1.81 \pm 0.35$. The error bars indicate the s.d.; $\mathrm{n}=3$ independent experiments.

Supplementary Figure 5. MlaFk47REDB displays no flippase activity. Kinetic fluorescence plot displaying the effect of dithionite quenching on the flippase activity assay corresponding to Fig. $3 \mathrm{~d}$.

Supplementary Figure 6. Lipid dependence of MlaFEDB flippase activity. A) MlaFEDB:NBD-PG flippase assay corresponding to Fig. 4 showing the effect of dithionite quenching on fluorescence levels. B) Kinetic fluorescence plot displaying the effect of dithionite quenching on the MlaFEDB:TopFluorCL flippase activity assay corresponding to Fig. 4.

Supplementary Figure 7. MlaC-Apo affects the transbilayer lipid distribution of MlaFEDB proteoliposomes. Graph corresponding to Fig. 5a showing changes in fluorescence in response to the dithionite quenching of MlaFEDB:NBD-PE proteoliposomes in response to incubation with either MlaC-Apo or MlaC-PL.

Supplementary Figure 8. Outer leaflet labelling of MlaFEDB proteoliposomes with NBD-PE. MlaFEDB reconstituted into E.coli polar lipid liposomes was incubated with $0.3 \%$ (w/w) NBD-PE in ethanol, resulting in incorporation into the outer leaflet of the proteoliposome only. Through dithionite quenching the levels of NBD- 
bioRxiv preprint doi: https://doi org/10.1101/2020.06.06.138008. this version posted June 6, 2020. The copyright holder for this preprint (which was not certified by peer review) is the author/funder, who has granted bioRxiv a license to display the preprint in perpetuity. It is made available under aCC-BY-NC-ND 4.0 International license.

PE in the respective leaflets of these proteoliposomes was assessed and compared to that of MlaFEDB proteoliposomes with NBD-PE distributed across both leaflets. 95\% of NBD-PE in MlaFEDB:NBD-PE ${ }^{\text {OUTER }}$ proteoliposomes was found in the outer leaflet. This was compared to MlaFEDB:NBD-PE ${ }^{\text {BOTH }}$ proteoliposomes in which NBD-PE was seen to be distributed relatively evenly across both leaflets.

Supplementary Figure 9. Raw image corresponding to figure 1A.

Supplementary Figure 10. Raw images corresponding to figure 3. A) Complete image of TLC plate corresponding to figure 3B. B) Raw SDS-PAGE image relating to figure 3C.

Supplementary Figure 11. Raw images corresponding to figure 5. A) Complete image of TLC plate corresponding to figure 4B. B) Raw TLC image relating to figure 4C.

Supplementary Figure 12. Raw image corresponding to supplementary figure 1A.

Supplementary Figure 13. Raw image corresponding to supplementary figure 4A. 
bioRxiv preprint doi: https://doi.org/10.1101/2020.06.06.138008: this version posted June 6.2020 . The copvriaht holder for this preprint (which was not certified by peer review) is the author/funder, who has granted bioRxiv a license to display the preprint in perpetuity. It is made available under aCC-BY-NC-ND 4.0 International license.

A.

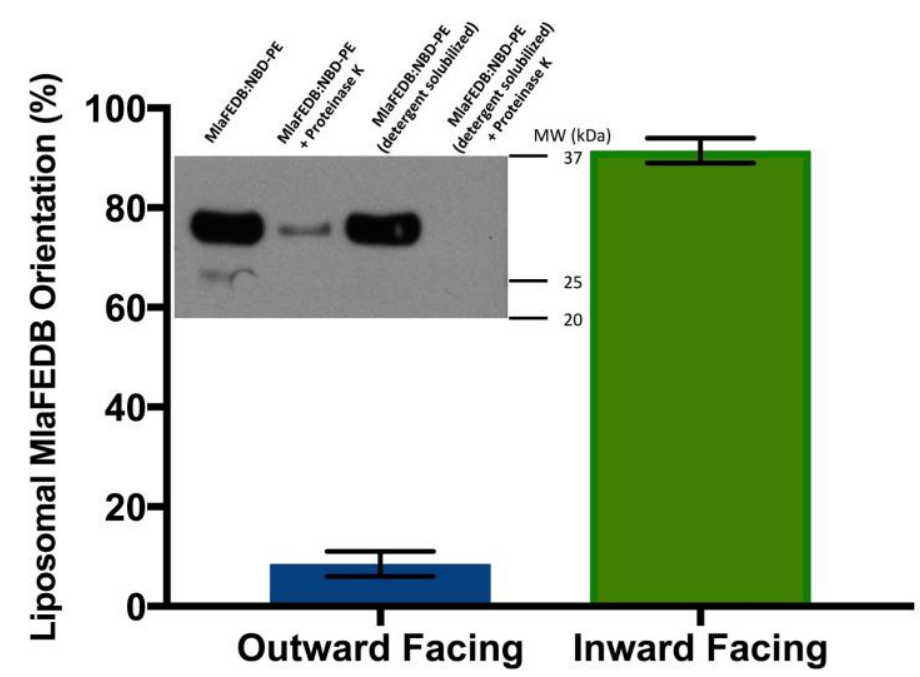

B.
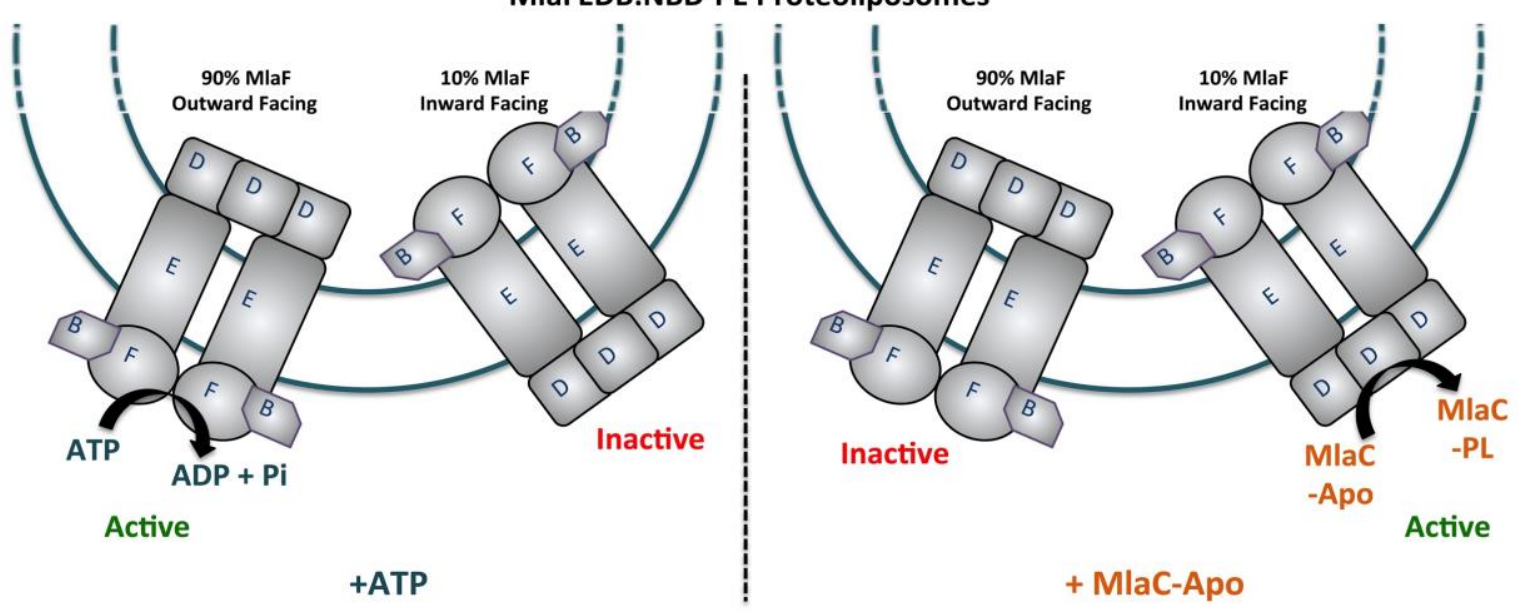

+ MlaC-Apo

Figure 1. 
bioRxiv preprint doi: https://doi org/10.1101/2020.06 06 138008; this version posted June 6, 2020. The copyright holder for this preprint (which was not certified by peer review) is the author/funder, who has granted bioRxiv a license to display the preprint in perpetuity. It is made available under aCC-BY-NC-ND 4.0 International license.
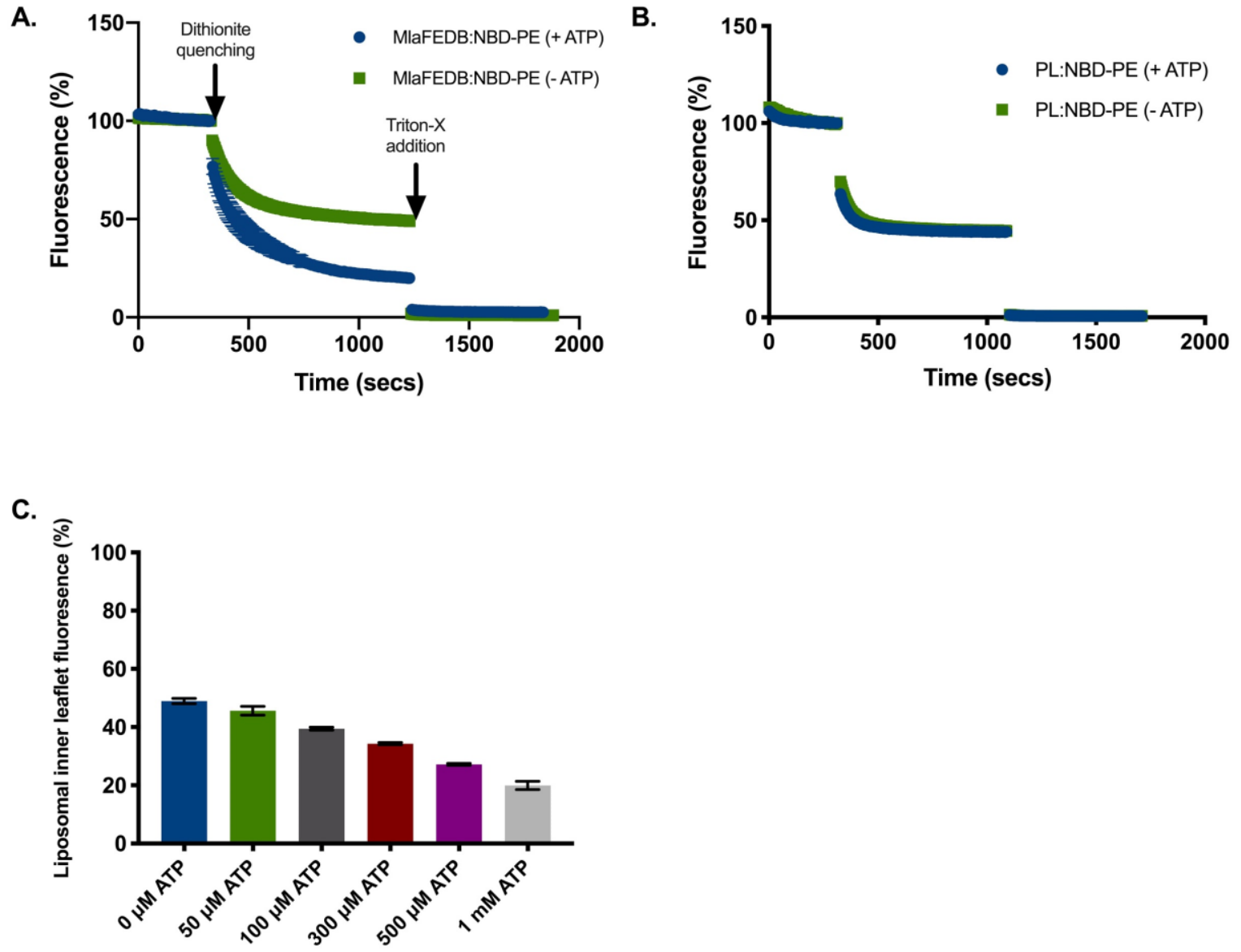

Figure 2. 
A.

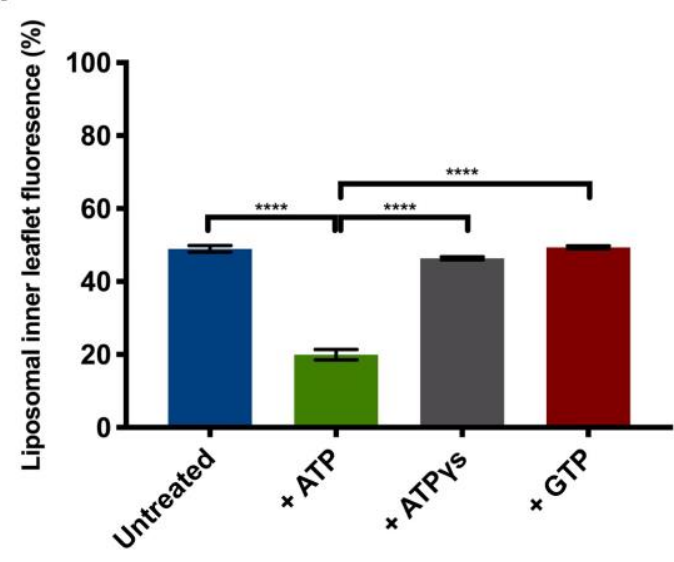

C.

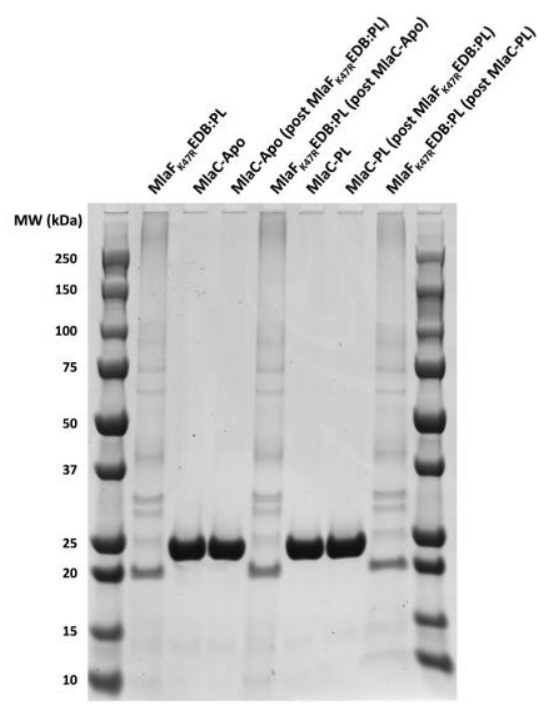

B.

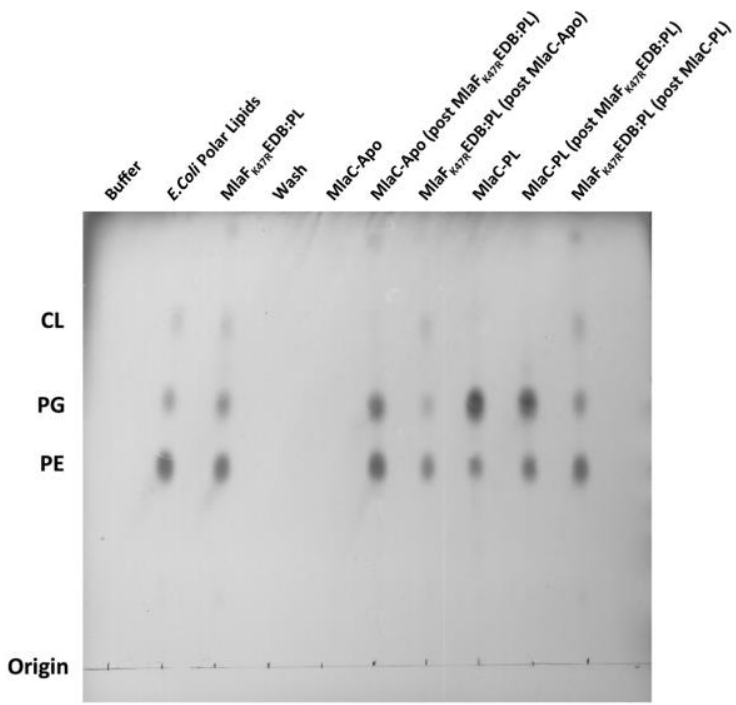

D.

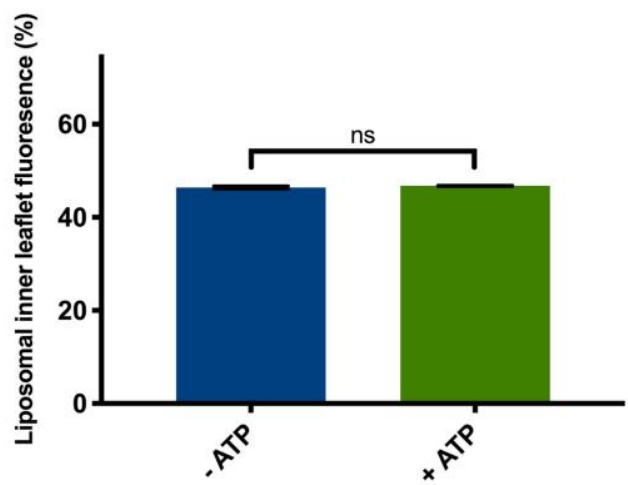

Figure 3. 


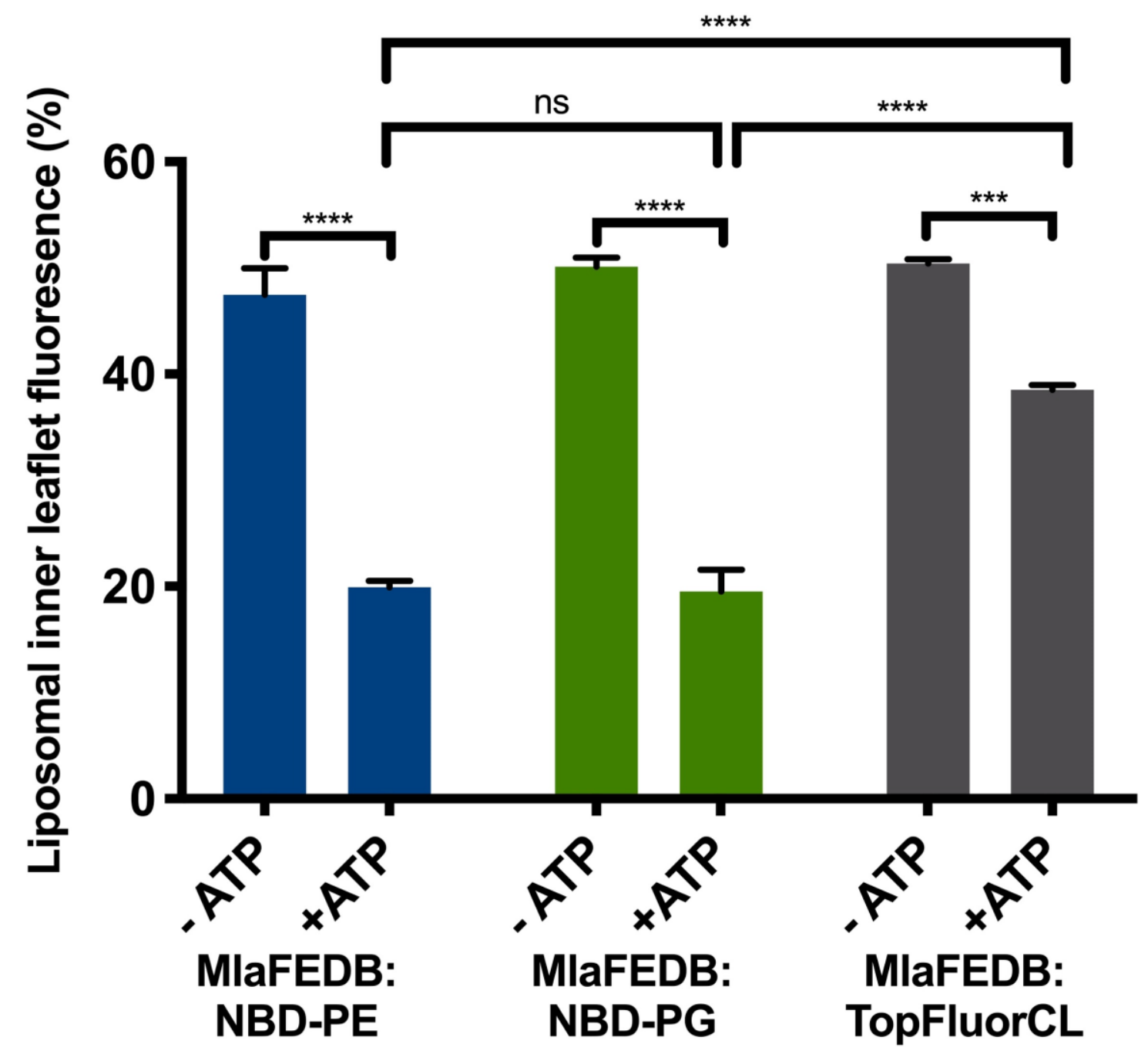

Figure 4. 
bioRxiv preprint doi: https://doi org/10.1101/2020.06 06.138008. this version posted June 6, 2020. The copyright holder for this preprint (which was not certified by peer review) is the author/funder, who has granted bioRxiv a license to display the preprint in perpetuity. It is made available under aCC-BY-NC-ND 4.0 International license.

A.

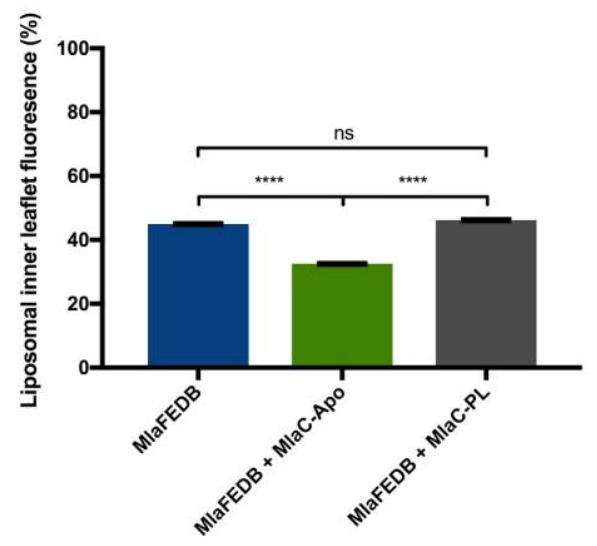

B.

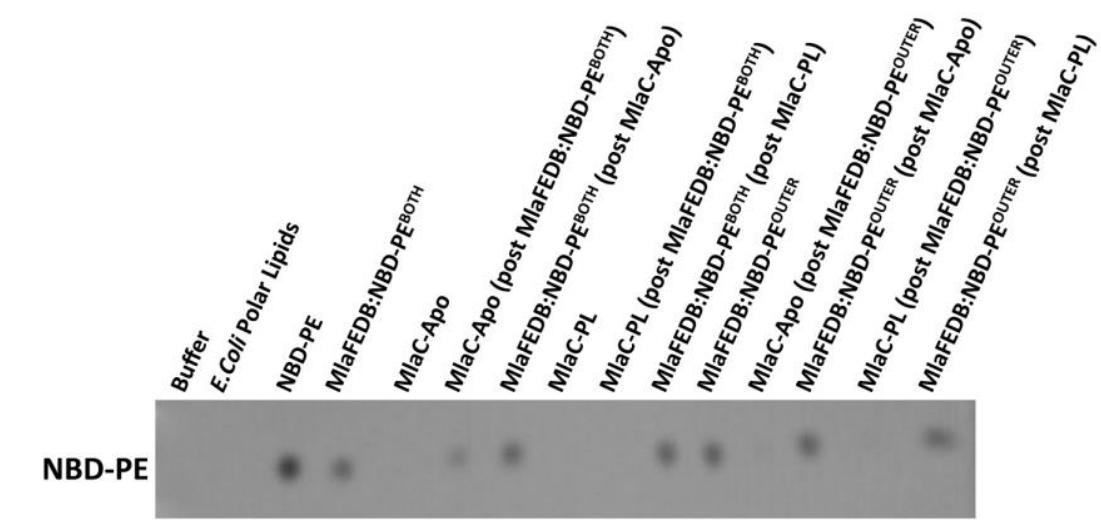

C.

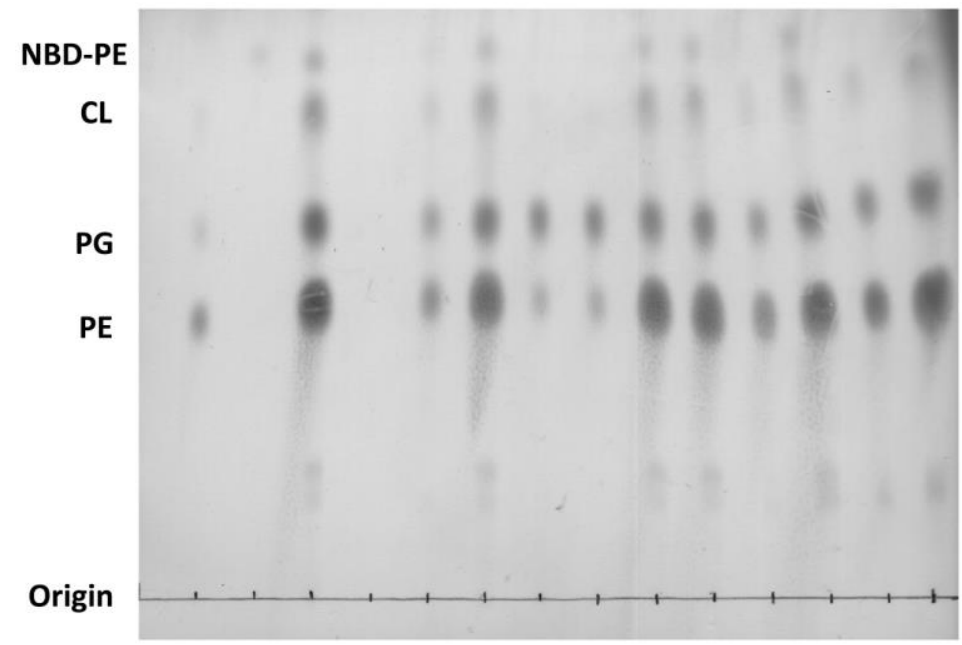

Figure 5. 

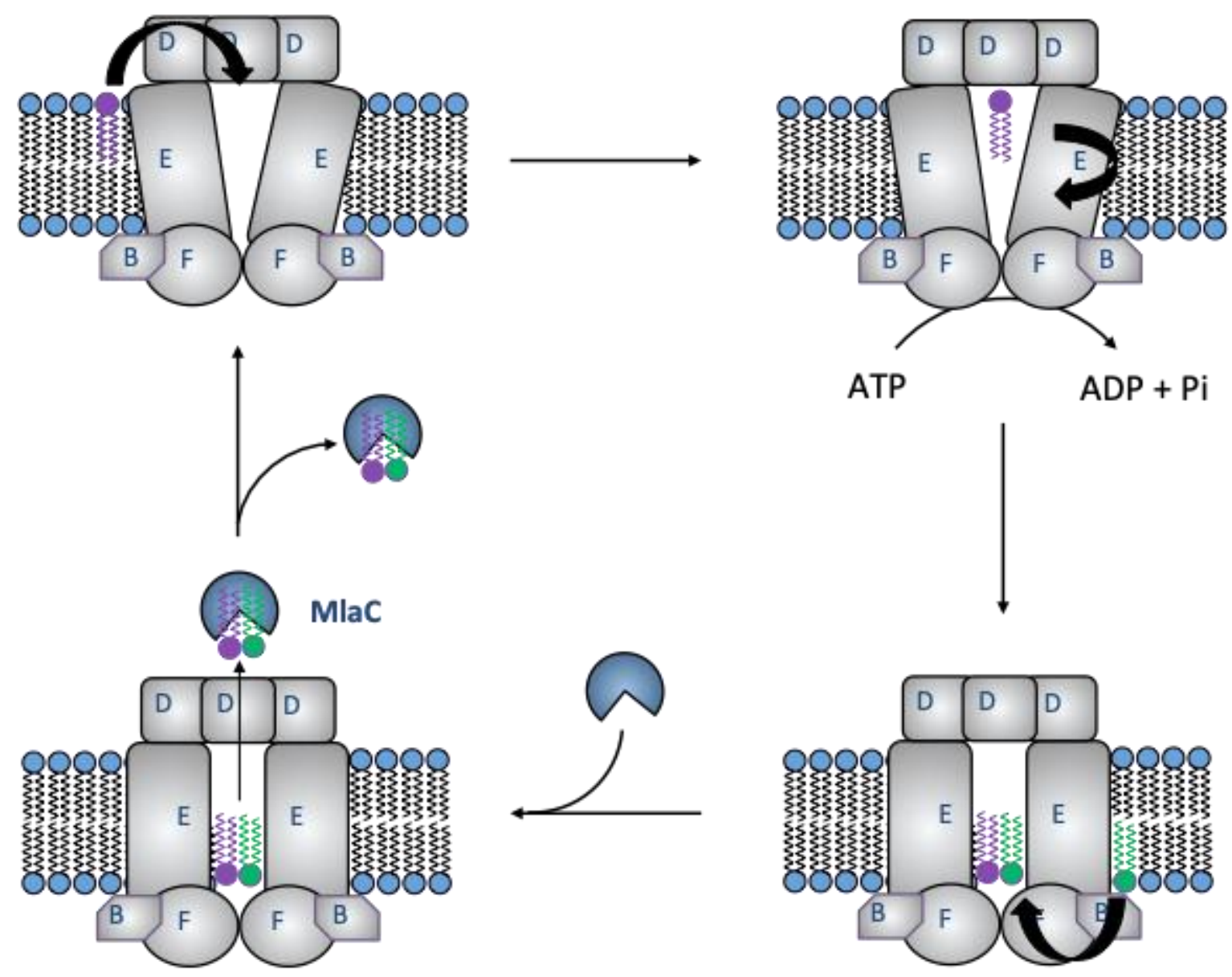

Figure 6. 
A.

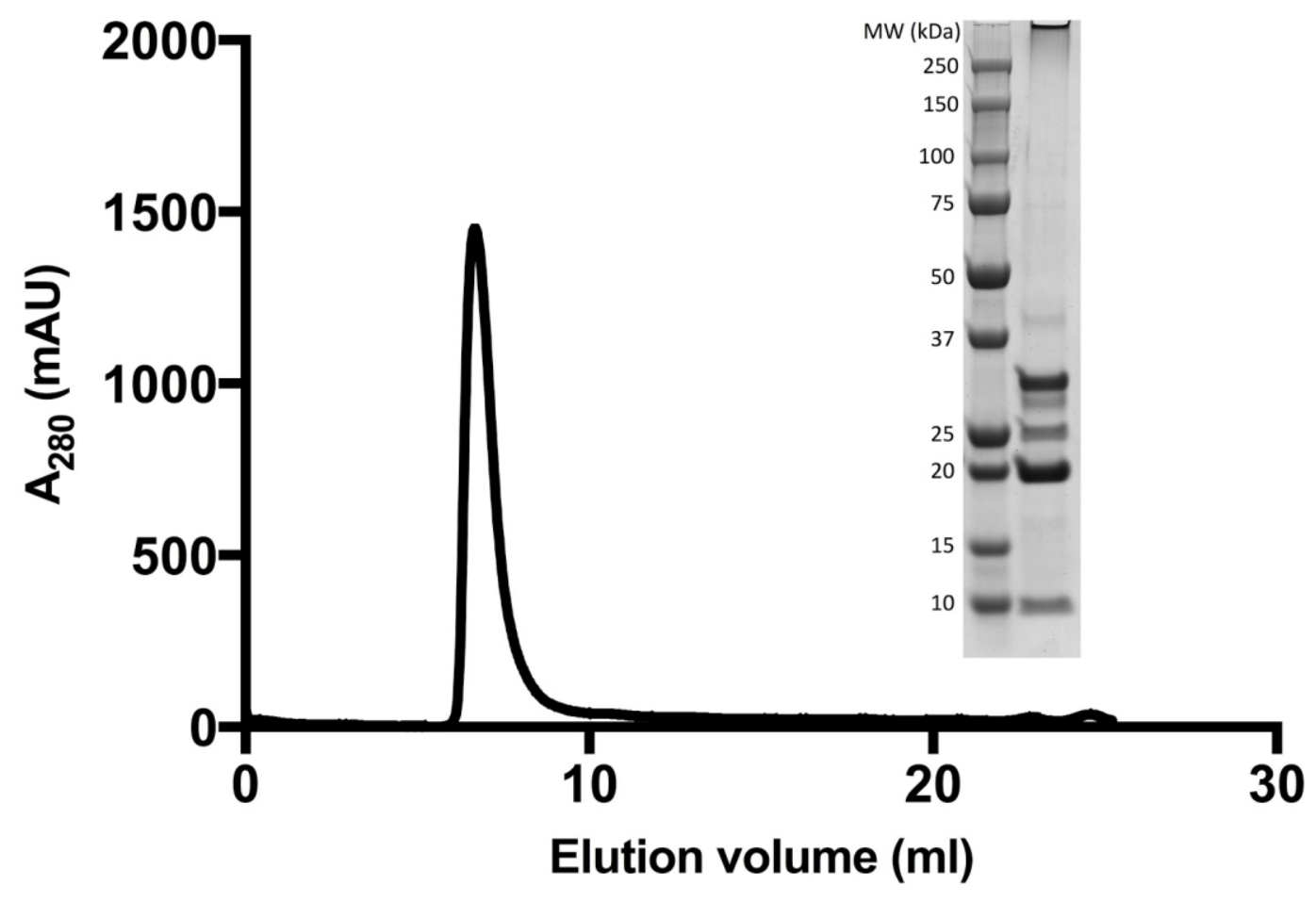

B.

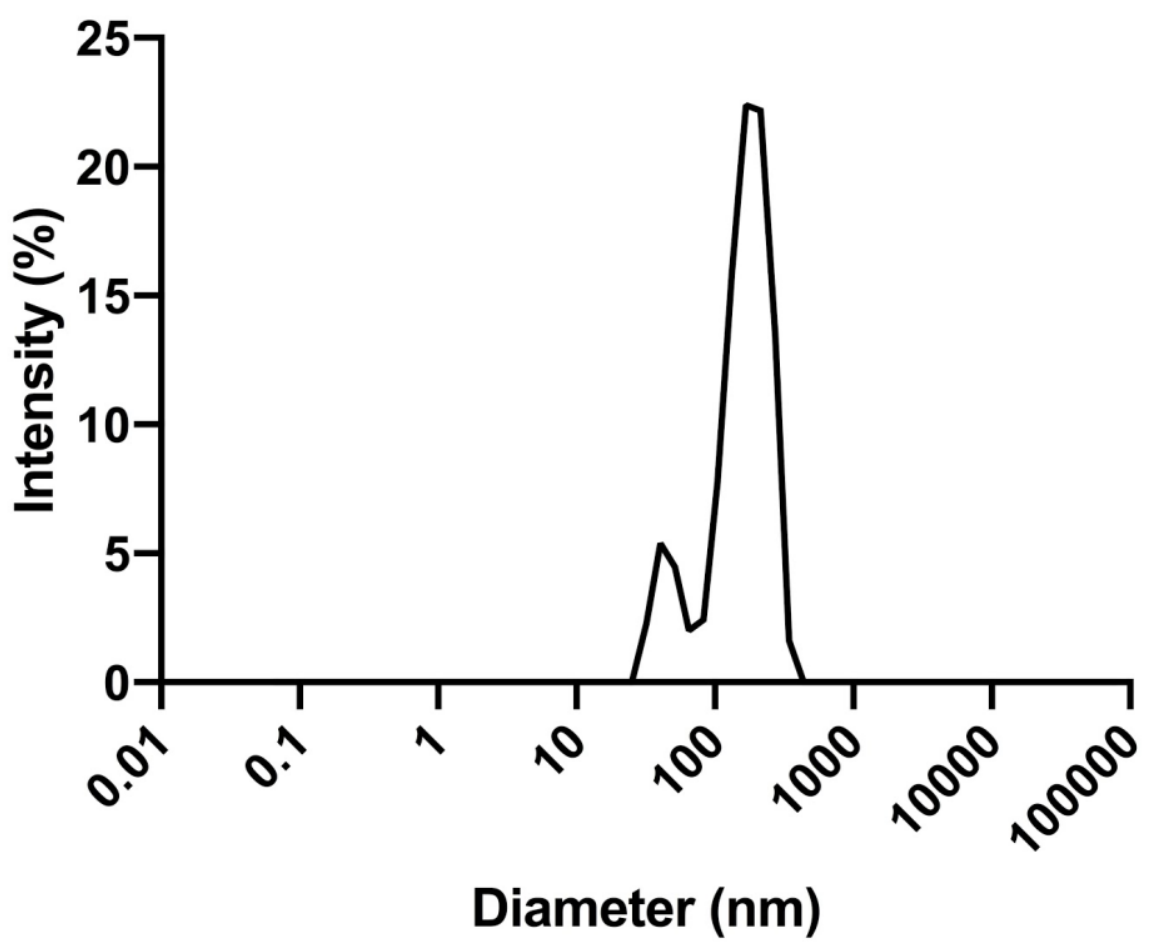

Supplementary Figure 1. 


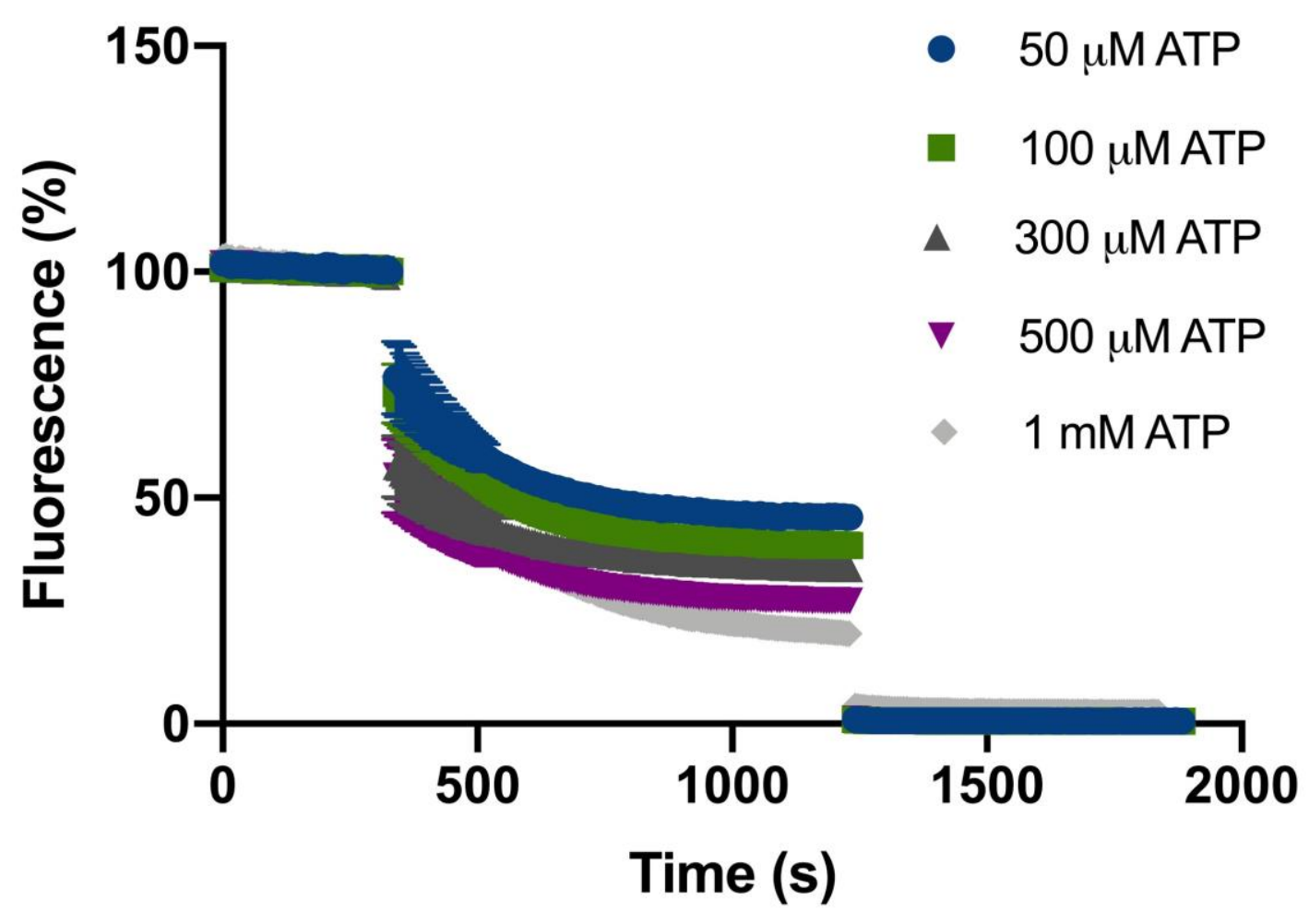

Supplementary Figure 2.

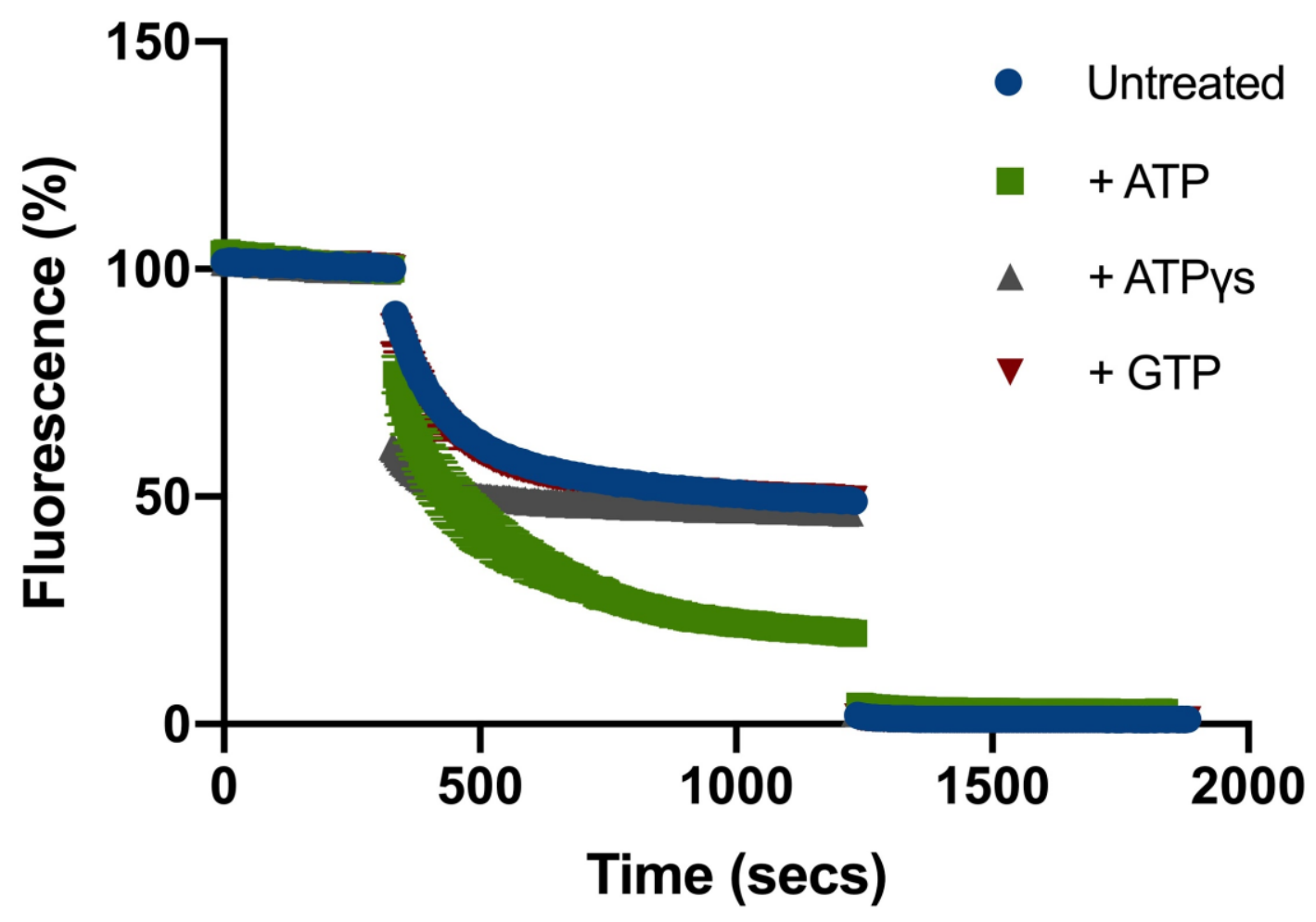


Supplementary Figure 3.
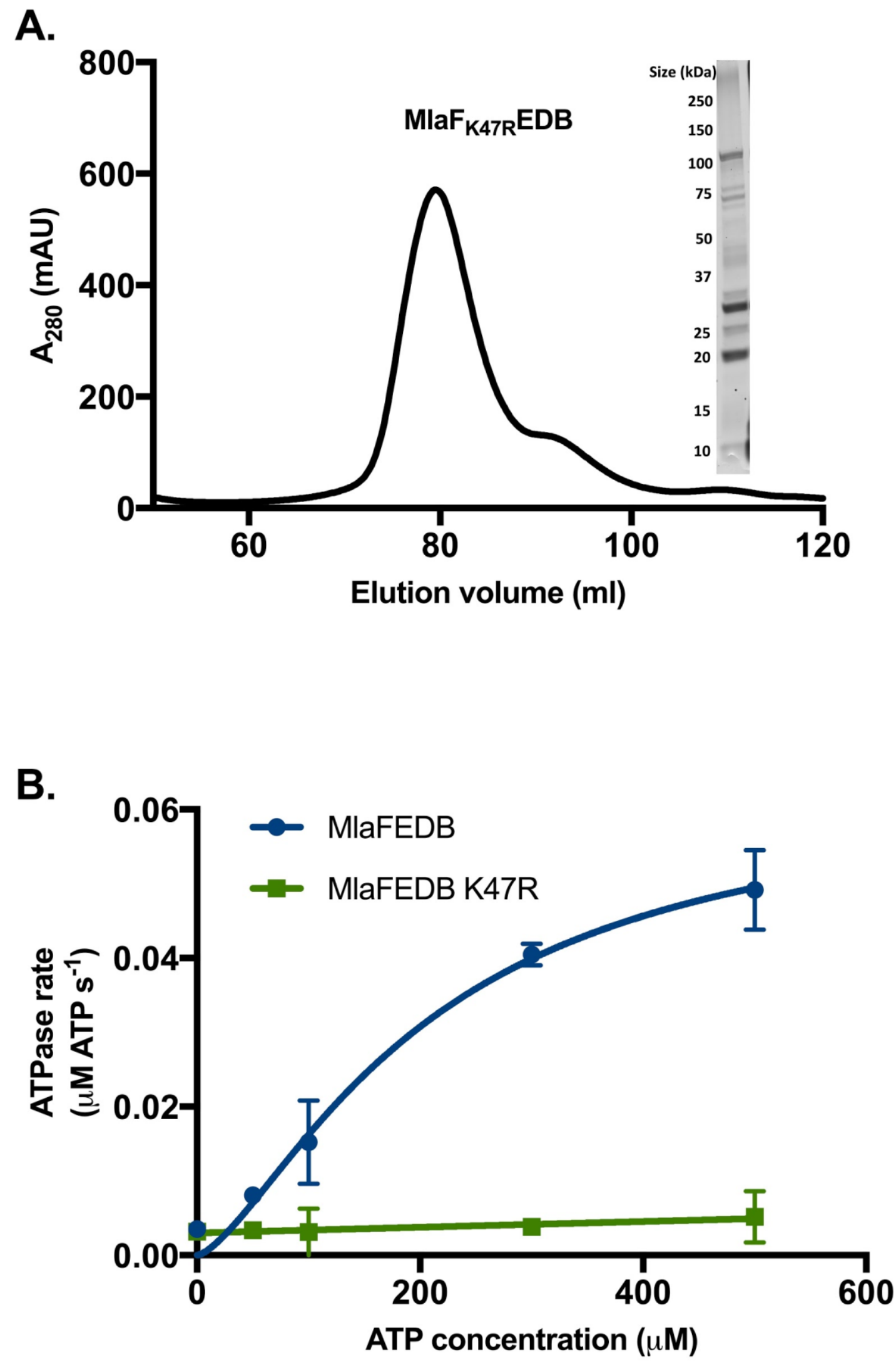
Supplementary Figure 4.

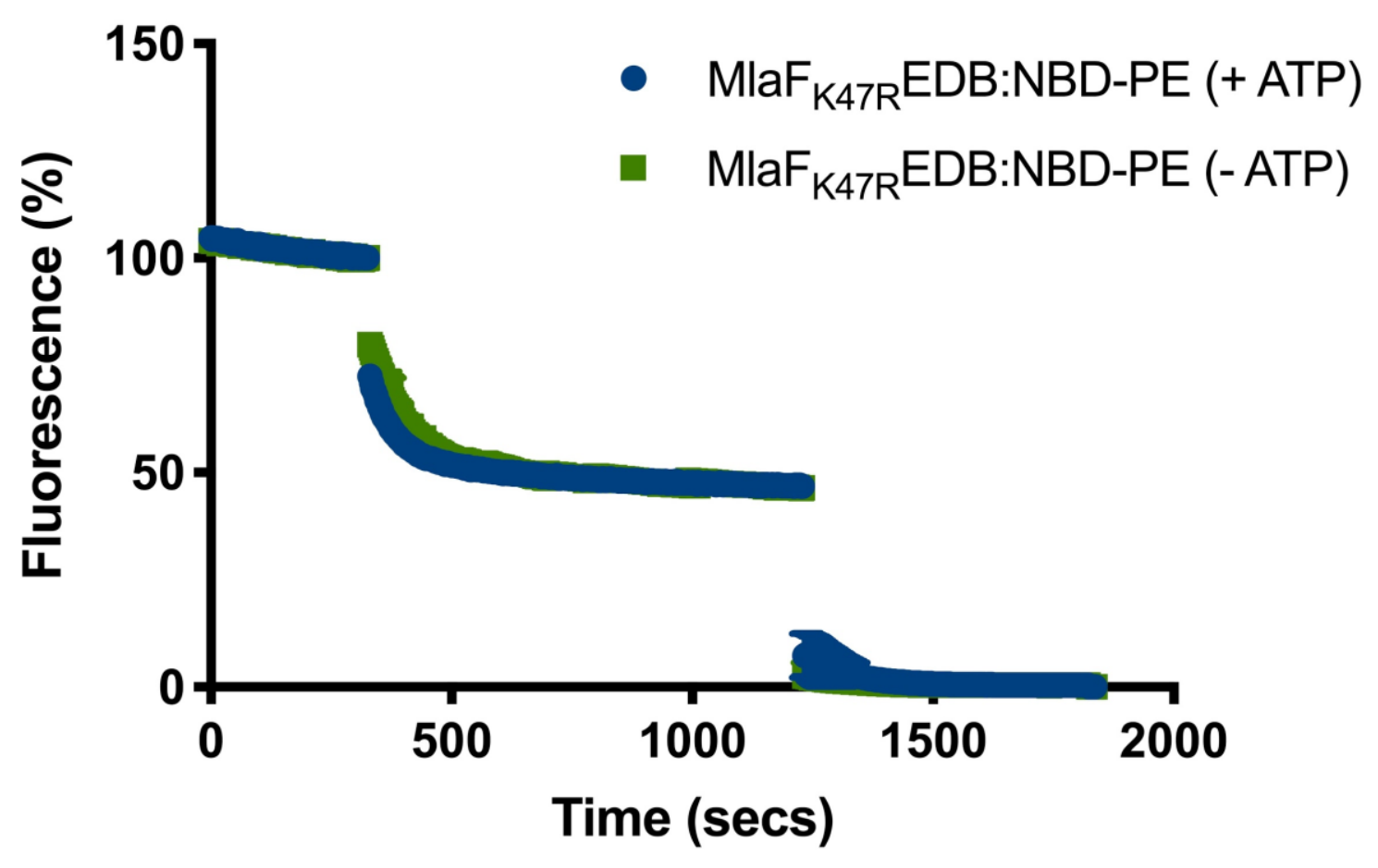

Supplementary Figure 5. 
A.

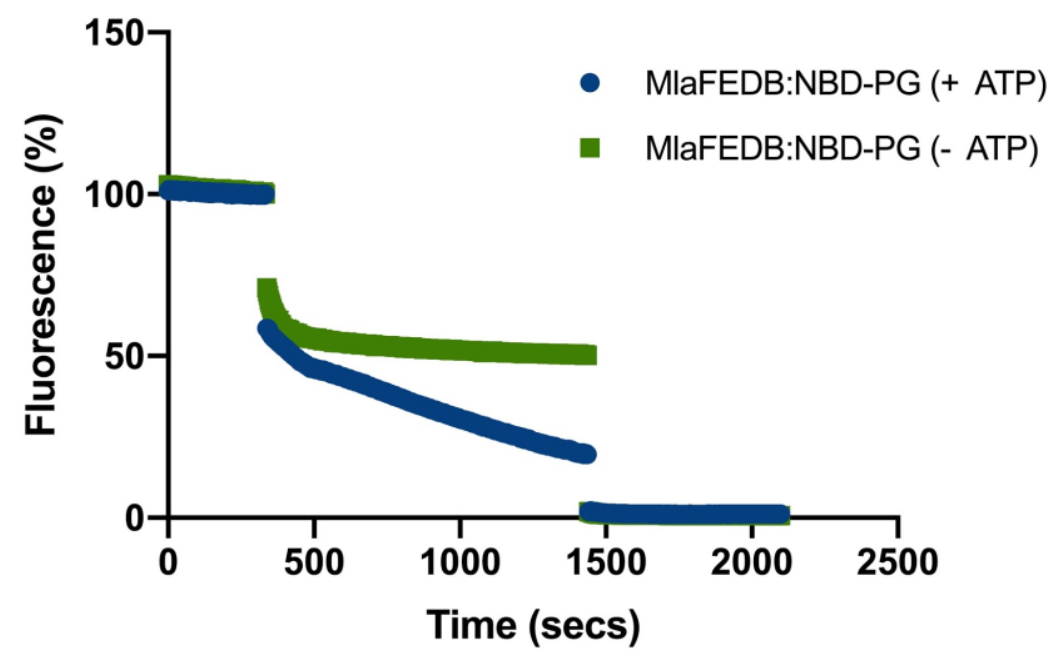

B.

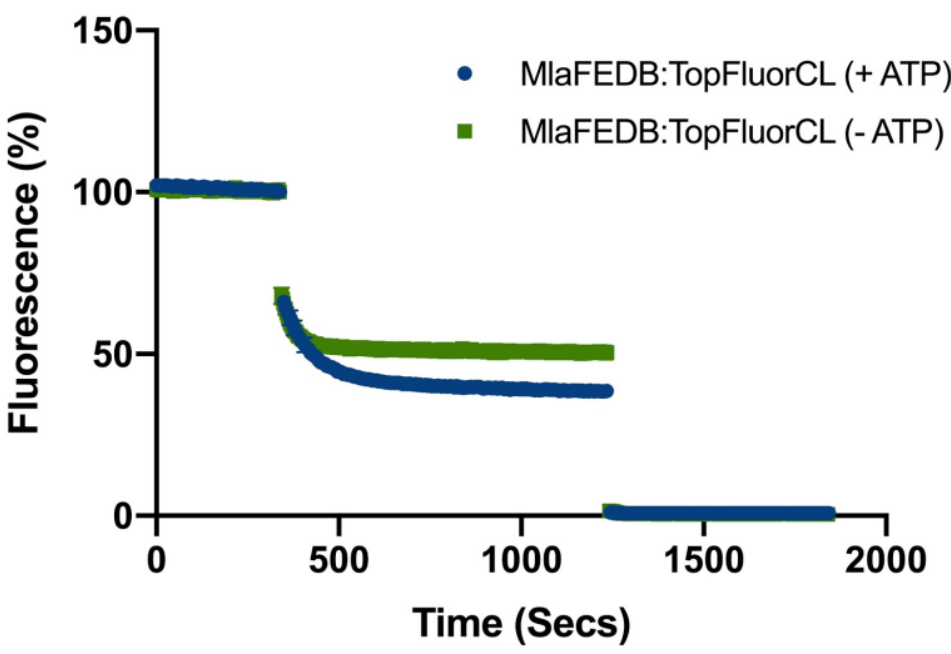

Supplementary Figure 6. 


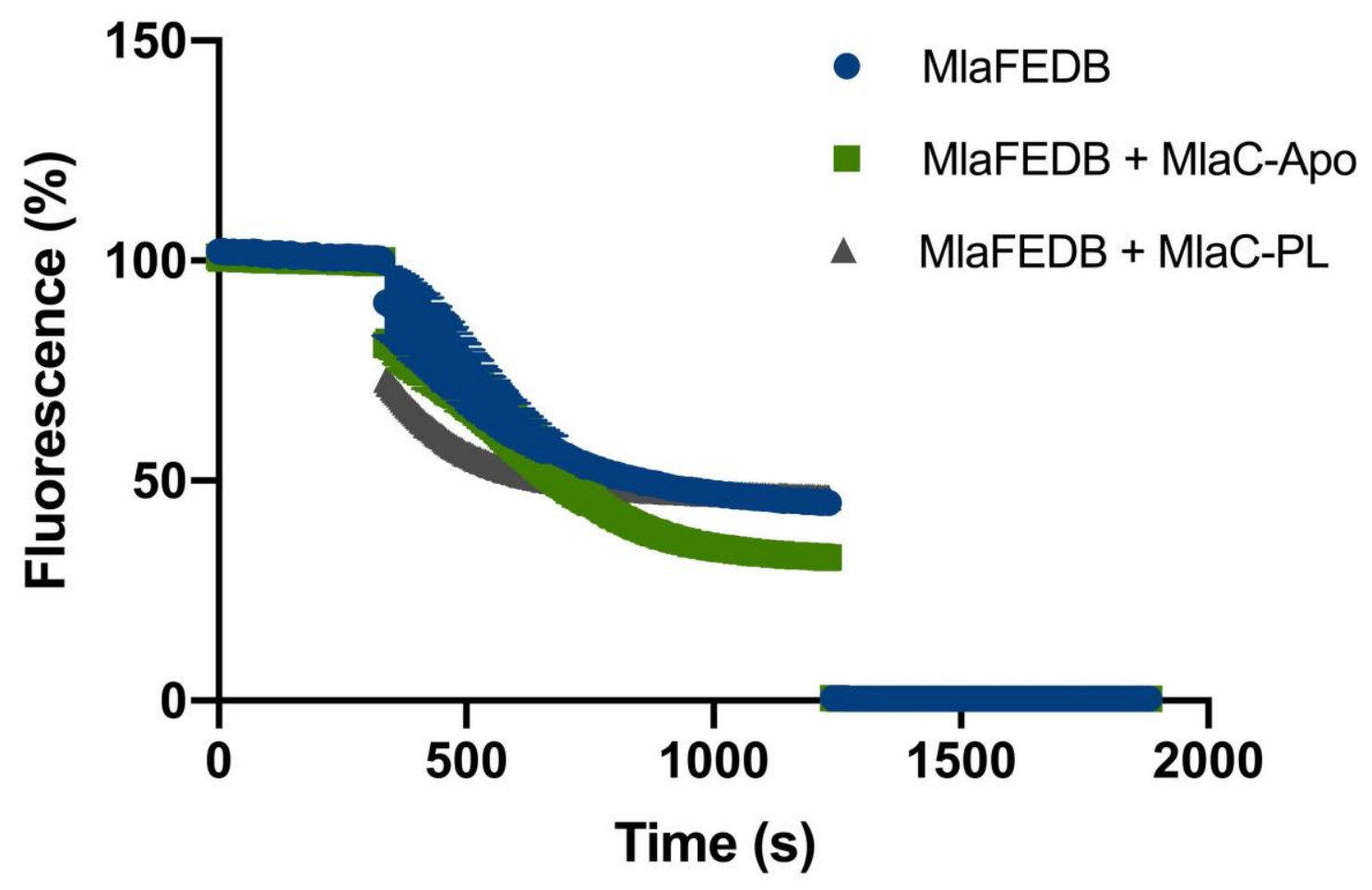

Supplementary Figure 7.

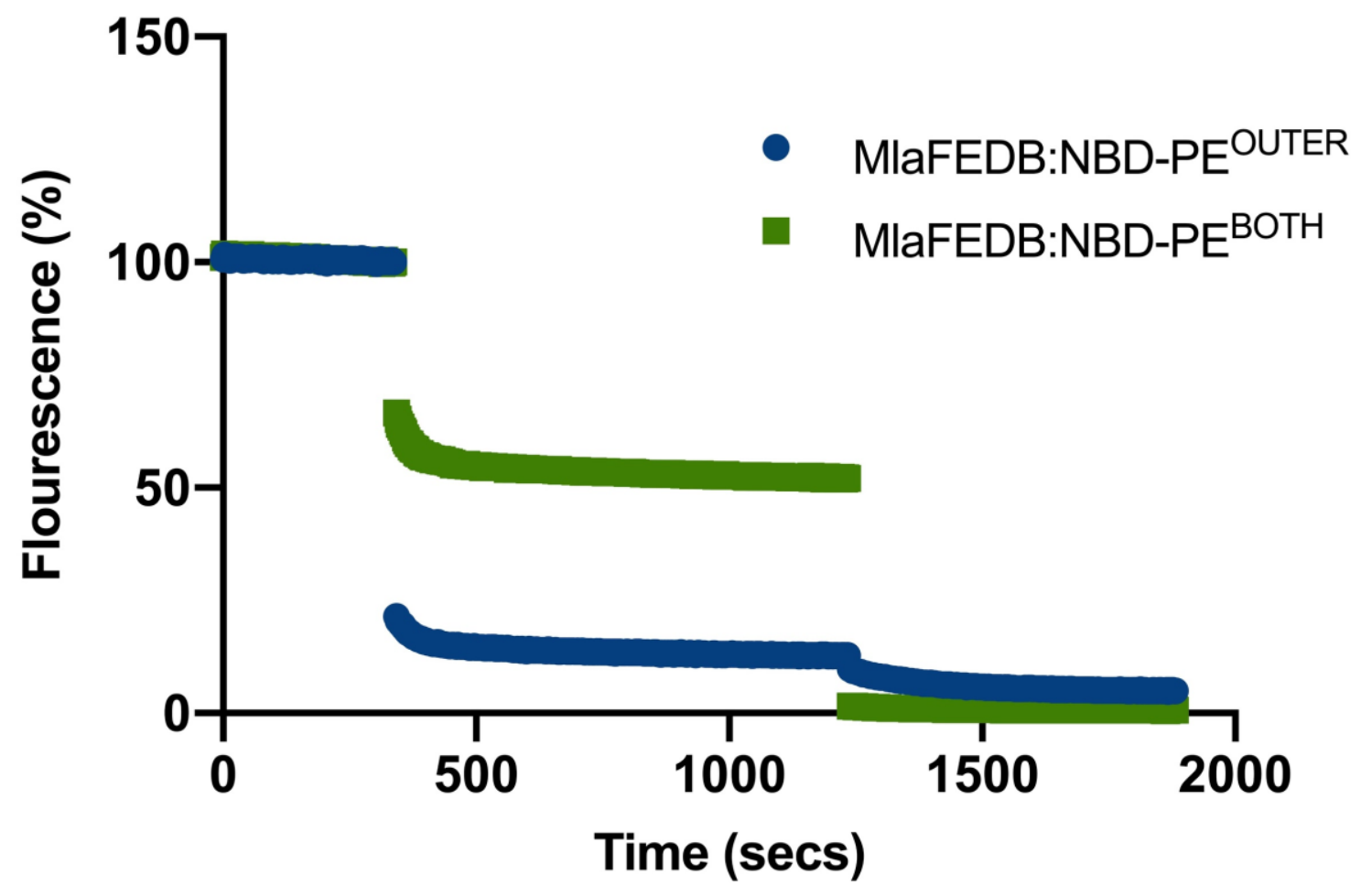


bioRxiv preprint doi: https://doi org/10.1101/2020 06 06.138008; this version posted June 6, 2020. The copyright holder for this preprint (which was not certified by peer review) is the author/funder, who has granted bioRxiv a license to display the preprint in perpetuity. It is made available under aCC-BY-NC-ND 4.0 International license.

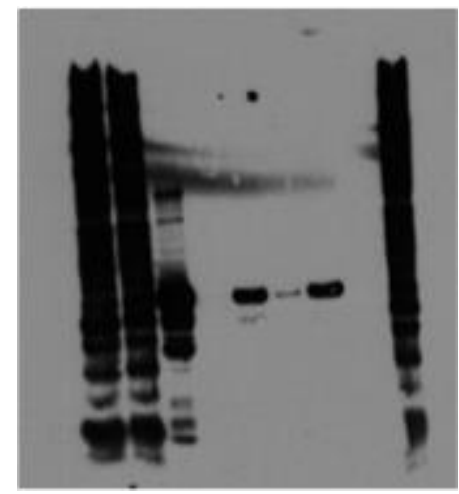

\section{Supplementary Figure 8.}

Supplementary Figure 9. 
bioRxiv preprint doi: https://doi.org/10.1101/2020.06.06.138008; this version posted June 6, 2020. The copyright holder for this preprint (which was not certified by peer review) is the author/funder, who has granted bioRxiv a license to display the preprint in perpetuity. It is made available under aCC-BY-NC-ND 4.0 International license. 
bioRxiv preprint doi: https://doi org/10.1101/2020.06 06.138008; this version posted June 6, 2020. The copyright holder for this preprint (which was not certified by peer review) is the author/funder, who has granted bioRxiv a license to display the preprint in perpetuity. It is made available under aCC-BY-NC-ND 4.0 International license.

A.

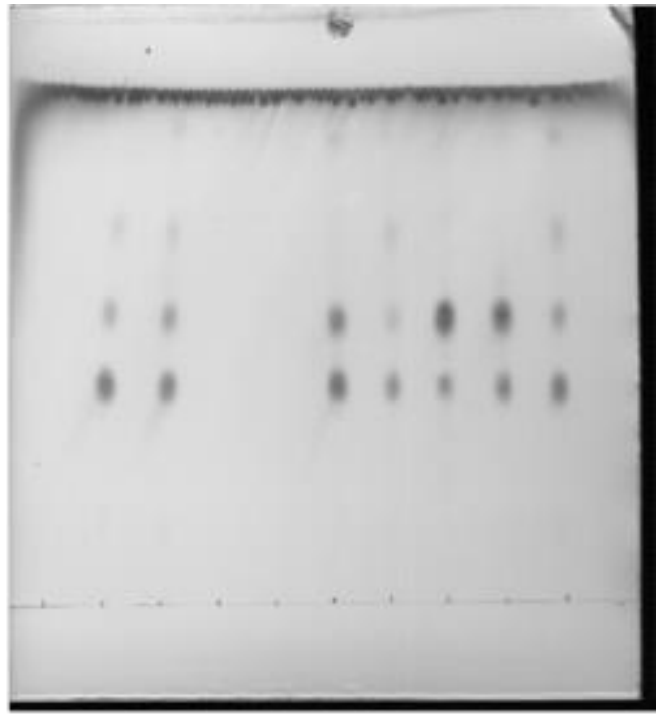

B.

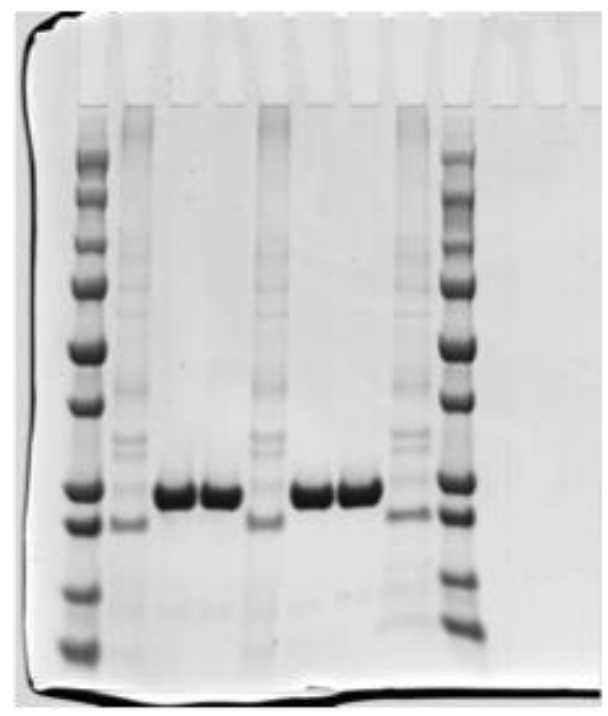

Supplementary Figure 10. 
bioRxiv preprint doi: https://doi org/101101/2020 06 06.138008; this version posted June 6, 2020. The copyright holder for this preprint (which was not certified by peer review) is the author/funder, who has granted bioRxiv a license to display the preprint in perpetuity. It is made available under aCC-BY-NC-ND 4.0 International license.

A.

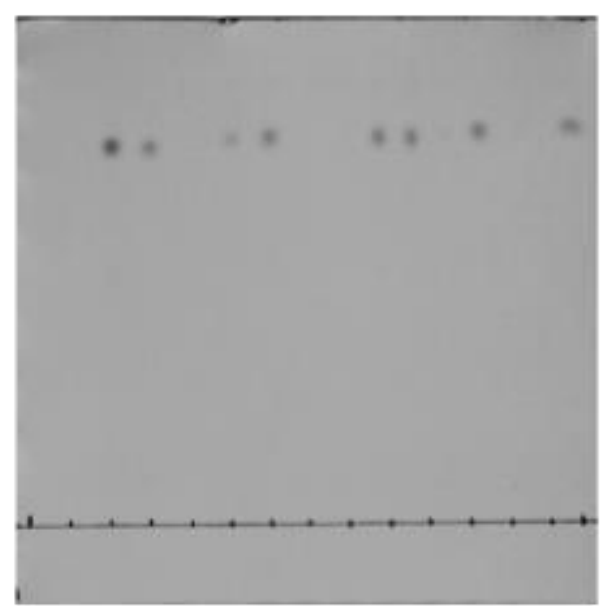

B.

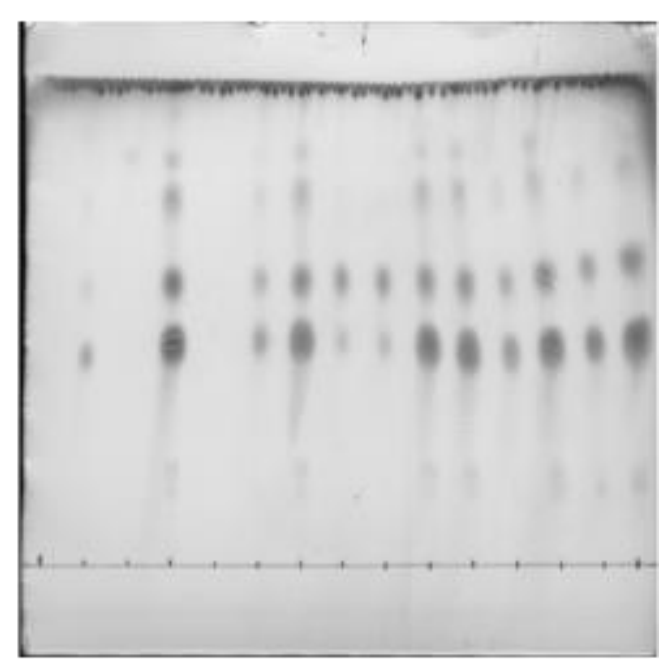

Supplementary Figure 11. 
bioRxiv preprint doi: https://doi org/10.1101/2020.06 06 138008. this version posted June 6, 2020. The copyright holder for this preprint (which was not certified by peer review) is the author/funder, who has granted bioRxiv a license to display the preprint in perpetuity. It is made available under aCC-BY-NC-ND 4.0 International license.

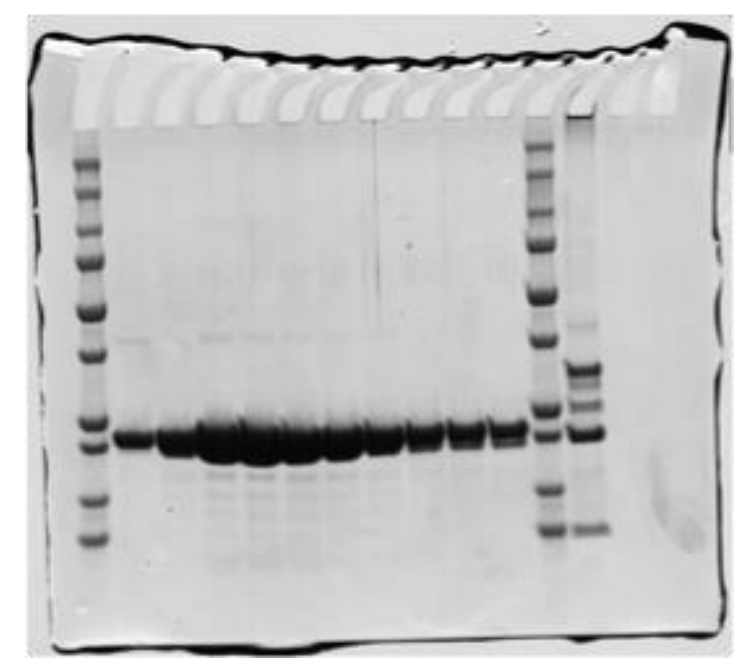

Supplementary Figure 12. 
bioRxiv preprint doi: https://doi org/10.1101/2020.06.06.138008. this version posted June 6, 2020. The copyright holder for this preprin (which was not certified by peer review) is the author/funder, who has granted bioRxiv a license to display the preprint in perpetuity. It is made available under aCC-BY-NC-ND 4.0 International license.

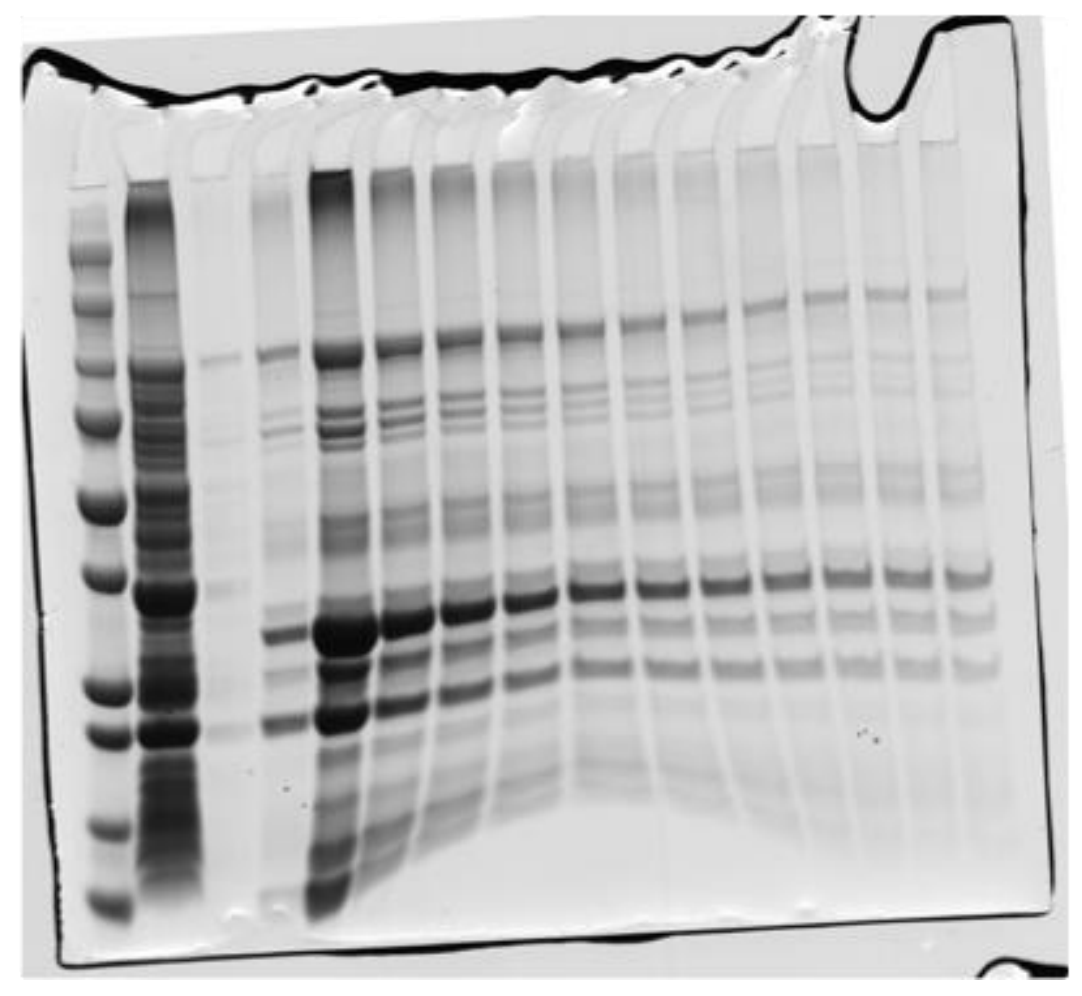

Supplementary Figure 13. 